

\title{
DISPERSION RELATIONS FOR SH WAVES ON A MAGNETOELECTROELASTIC HETEROSTRUCTURE WITH IMPERFECT INTERFACES
}

\author{
José A. Otero, Héctor Calas, Reinaldo Rodríguez-Ramos, \\ Julián Bravo-Castillero, Adair R. Aguiar and Guillermo Monsivais
}

\begin{abstract}
The dispersion relations of surface $\mathrm{SH}$ waves in an $\mathrm{A} / \mathrm{B} / \mathrm{A}$ heterostructure with magnetoelectroelastic properties and imperfect (electromagnetically permeable or absorbent, mechanically spring-type) bonding at the interfaces are obtained taking the geometric symmetry of the system into account. Consequently, the results for the symmetric and antisymmetric modes are presented. Different limit cases are considered. Numerical calculations for relevant realizations of the heterostructure are investigated for different values of the material parameter describing the assumed mechanically imperfect bonding. In all the studied cases, the propagation velocities of the $\mathrm{SH}$ waves increase for increasing values of this parameter and are limited by the velocities on the homogeneous phases A and B.
\end{abstract}

\section{Introduction}

The development of smart structures is currently receiving widespread attention owing to potential applications in several branches of engineering, such as in integrated control architecture with highly distributed sensors and actuators. More recently, applications can be found in the design of smart materials, where piezoelectric and piezomagnetic properties are involved. The problem of wave propagation in this type of material has been studied by different authors using different geometries. For instance, in [Alshits et al. 1994] the existence of localized acoustic waves was studied on the interface between two piezocrystals of arbitrary anisotropy. In [Pan 2001] an exact closed-form solution was derived for the static deformation of multilayered piezoelectric and piezomagnetic plates based on quasi-Stroh formalism and using the propagator matrix method. In [Pan and Heyliger 2002] the analytical method of [Pan 2001] was extended to the free vibration of three-dimensional, anisotropic linearly magnetoelectroelastic (MEE) simply supported, and multilayered rectangular plates. In [Wang et al. 2003] the state vector equations for three-dimensional, orthotropic, and linearly MEE media were derived. The solution of these equations is based on a mixed formulation, where the basic unknowns are not only the displacements, electrical potential, and magnetic potential, but also the stresses, electric displacements, and magnetic induction. Recently, [Chen et al. 2007] presented an analytical treatment for the propagation of harmonic waves in infinitely extended MEE multilayered plates based on the state vector approach.

The authors wish to acknowledge the National Council for Scientific and Technological Development (CNPq), Proc. No. 450462/2009-9, the National Program of Basic Sciences, CITMA, Cuba, PNCIT IBMFQC 0709-0000, CoNaCyT/México project No. 82474 and DGAPA UNAM/México project IN119509, for their support of this research. JBC is also grateful for the support provided by CGCI/CAPES/Brazil project No. 0452-11/2010.

Keywords: piezoelectricity, piezomagnetism, magnetoelectroelasticity, dispersion curve, wave propagation, imperfect contact. 
The existence of interfaces is a usual feature of both composite materials and structures. Depending on the materials involved and how they are put together (for example, by different glues), a thin layer with its own material properties, which are noticeably different from those of the participating materials, may manifest itself at the interface. The simplest description of the mechanical behavior of an interface is modeled as a layer that geometrically has a zero thickness but still possesses elasticity and interface elastic strain energy, for example, the shear-lag model of [Cheng et al. 1996; Handge 2002], in which the tangential displacement at an interface is allowed to be different from both sides of the interface to account for the deformation of the interface layer. At the same time, the inertia of the interface is neglected so that the traction across the interface is continuous and is proportional to the displacement jump across the interface. In this description, an interface is essentially considered as having many massless springs. A similar description can also be applied in the normal direction of an interface.

In practice, due to various causes such as microdefects, diffusion impurity, damage, weak bonding, et cetera, two dissimilar materials cannot be perfectly bonded, and an interface or transition with thickness in the range of 30-240 nm exists across the contact surfaces [Termonia 1990]. These transition zones weaken the interfacial continuity, and further affect the performance of the heterogeneous structure; in particular, the interfacial characteristics [Margetan et al. 1992; Wu et al. 2002]. An adequate description of such an interface is quite complex. A simplified model consists of considering an imperfect interface with vanishing thickness, at which the stresses can transfer continuously, but the displacements have a jump. In general, linear spring relations are assumed between the normal stress and the displacement jump in the normal direction and between the tangential stress and the displacement jump in the tangential direction [Hashin 1991; Klarbring and Movchan 1998; Zhong et al. 2009]. Using this modeling, the effects of interfacial imperfection on waves propagating in an isotropic elastic bimaterial have been analyzed in [Rokhlin and Wang 1991; Huang and Rokhlin 1992; Deng 2006]. For a class of smart materials, when the bonding interface is fully debonded, a small gap appears; the gap wave propagation has been investigated in [Li and Yang 2006]. The effects of an imperfectly bonded interface of piezoelectric waves near such interface have been studied in [Fan et al. 2006b] and [Yang et al. 2006], respectively, for two bonded piezoelectric materials. For an imperfect interface of a two-phase piezoelectric/piezomagnetic structure, interfacial shear horizontal waves propagating along an imperfect interface have been formulated in [Huang et al. 2009].

Shear-lag type interface models have been mainly used in static analysis. Recently, theoretical analysis was given on vibrations and waves propagating in structures with interfaces described by the shear-lag model [Chen et al. 2004; Yang et al. 2006].

The present work is motivated by recent contributions dealing with the effects of the elastic interface on vibration and wave propagation in composites. For instance, in [Fan et al. 2006a; 2006b; Chen et al. 2008; Melkumyan and Mai 2008] waves propagating in composite structures with imperfectly bonded interfaces were studied. Here, we propose a generalization of [Calas et al. 2008] to the case of imperfect contact conditions. In that study, the authors considered the case of perfect bonding at the interfaces of dissimilar media. In the present contribution, the interface is modeled by the shear-lag model for imperfect bonding. Here, the behavior of stationary SH waves in a heterostructure with MEE materials with imperfect bonding on the interface is studied. In particular, the case of permeable and absorbent interfaces is discussed in detail. The governing system of partial differential equations is solved using the geometric symmetry of the heterostructure and considering the superficial acoustic wave. Solutions 
can be separated into symmetric and antisymmetric parts. The dispersion curves and the influence of the imperfection at the interface are shown for some cases.

The work is organized as follows: in Section 2 the governing equations of SH waves propagating in a MEE homogeneous medium with $6 \mathrm{~mm}$ hexagonal symmetry are presented. Section 3 is devoted to the formulation of the mean problem of an A/B/A heterostructure considering two types of imperfect interfacial conditions, and to the derivation of the symmetric and antisymmetric solutions of a related auxiliary problem. In Section 4, the dispersion relations are obtained, and different limit cases are described and shown to be in agreement with others reported in the literature. In Section 5 numerical examples are presented and discussed. Finally, some concluding remarks are given in Section 6.

\section{Wave equation for the $\mathrm{SH}$ mode}

Consider a MEE material with $6 \mathrm{~mm}$ hexagonal symmetry exhibiting magnetization and polarization in the $z$-axis direction of an $x y z$ Cartesian coordinate system. The $x y$-plane is the isotropy plane. In this type of material, the SH wave is described by a system of three coupled partial differential equations with three unknowns: the $z$-component of the elastic displacement $u_{z}$, the in-plane electric potential $\varphi$, and the in-plane magnetic potential $\psi$ (see, for instance, [Melkumyan 2007; Wang et al. 2007; Calas et al. 2008; Huang and Li 2010]), that is,

$$
\begin{aligned}
c \nabla^{2} u+e \nabla^{2} \varphi+f \nabla^{2} \psi & =\rho \frac{\partial^{2} u}{\partial t^{2}}, \\
e \nabla^{2} u-\varepsilon \nabla^{2} \varphi-g \nabla^{2} \psi & =0 \\
f \nabla^{2} u-g \nabla^{2} \varphi-\mu \nabla^{2} \psi & =0,
\end{aligned}
$$

with $\nabla^{2} \equiv \partial^{2} / \partial x^{2}+\partial^{2} / \partial y^{2}, u \equiv u_{z}$, and where $c \equiv c_{44}, e \equiv e_{15}, f \equiv f_{15}, \varepsilon \equiv \varepsilon_{11}, \mu \equiv \mu_{11}$, and $g \equiv g_{11}$ are the elastic, piezoelectric, piezomagnetic, dielectric permittivity, magnetic permeability, and magnetoelectric coefficients, respectively. In this study, the in-plane elastic displacements are $u_{x}=u_{y}=0$. Equations (2-1)-(2-3) describe the motion of a SH wave in a homogeneous material and, therefore, depend only on $(x, y, t)$.

The stress component $T \equiv T_{z y}$, the electric displacement $D \equiv D_{y}$, and the magnetic induction $B \equiv B_{y}$ are related to $u, \varphi$, and $\psi$ by

$$
T=c \frac{\partial u}{\partial y}+e \frac{\partial \varphi}{\partial y}+f \frac{\partial \psi}{\partial y}, \quad D=e \frac{\partial u}{\partial y}-\varepsilon \frac{\partial \varphi}{\partial y}-g \frac{\partial \psi}{\partial y}, \quad B=f \frac{\partial u}{\partial y}-g \frac{\partial \varphi}{\partial y}-\mu \frac{\partial \psi}{\partial y} .
$$

Due to the linearity of the Laplacian operator $\nabla^{2}$, it is possible to write (2-2) and (2-3) in the form

$$
\nabla^{2} \tilde{\varphi}=0, \quad \nabla^{2} \tilde{\psi}=0,
$$

where $\tilde{\varphi}$ and $\tilde{\psi}$ are auxiliary potential functions defined by

$$
\tilde{\varphi}=\varphi-\frac{e}{\varepsilon} u+\frac{g}{\varepsilon} \psi, \quad \tilde{\psi}=\psi-\frac{f}{\mu} u+\frac{g}{\mu} \varphi .
$$

Solving (2-6) for the functions $\varphi$ and $\psi$, we find

$$
\varphi=\chi \tilde{\varphi}+\frac{\bar{e}}{\varepsilon} u-\frac{\bar{g}}{\varepsilon} \tilde{\psi}, \quad \psi=\chi \tilde{\psi}+\frac{\bar{f}}{\mu} u-\frac{\bar{g}}{\mu} \tilde{\varphi},
$$


where

$$
\chi=\frac{\varepsilon \mu}{\varepsilon \mu-g^{2}}, \quad \bar{g}=g \chi, \quad \bar{e}=e \chi-\frac{f}{\mu} \bar{g}, \quad \bar{f}=f \chi-\frac{e}{\varepsilon} \bar{g} .
$$

Replacing the expressions given by (2-7) into (2-1), the typical wave motion equation for the mechanical displacement $u$ is given by

$$
\left(\nabla^{2}-\frac{1}{\bar{v}^{2}} \frac{\partial^{2}}{\partial t^{2}}\right) u=0
$$

where

$$
\bar{v}=\sqrt{\bar{c} / \rho}
$$

is the bulk shear wave speed of the MEE homogeneous medium and

$$
\bar{c}=c+e \bar{e} / \varepsilon+f \bar{f} / \mu
$$

is the magnetoelectroelastically stiffened elastic constant.

In the next section, a heterogeneous medium will be studied (see Figure 1), and (2-5) and (2-9) must be solved in three different homogeneous zones of the $x y$-plane. In order to determine $u, \tilde{\varphi}$, and $\tilde{\psi}$, suitable conditions should be employed. Moreover, it is convenient to express the constitutive relations (2-4) in the form

$$
T=\bar{c} \frac{\partial u}{\partial y}+\bar{e} \frac{\partial \tilde{\varphi}}{\partial y}+\bar{f} \frac{\partial \tilde{\psi}}{\partial y}, \quad D=-\varepsilon \frac{\partial \tilde{\varphi}}{\partial y}, \quad B=-\mu \frac{\partial \tilde{\psi}}{\partial y}
$$

\section{SH waves in MEE heterostructure}

In the $x y$-plane, we consider an infinite heterostructure $\mathrm{A} / \mathrm{B} / \mathrm{A}$ formed by two half-spaces $(y \leq-d / 2$ and $y \geq d / 2$ ) and an intermediate layer $(|y| \leq d / 2)$, as illustrated in Figure 1 . Two different homogeneous transversely isotropic MEE materials, with $6 \mathrm{~mm}$ symmetry, occupy regions A and $\mathrm{B}$. The magnetization

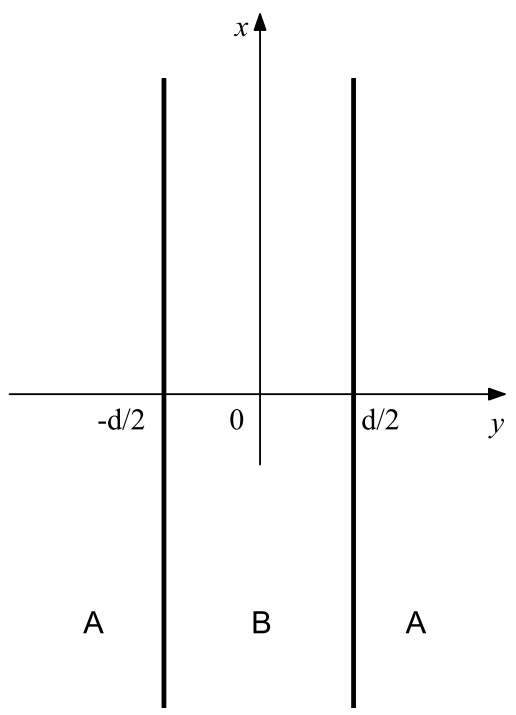

Figure 1. Scheme of a heterostructure A/B/A made of two magnetoelectroelastic materials. 
and polarization directions are supposed perpendicular to the $x y$-plane. Consequently, the equations presented in Section 2 must be satisfied in each one of the phases. We investigate the dispersion relations of SH-wave solutions $u, \varphi$, and $\psi$ of (2-1)-(2-3) for two types of imperfect conditions on $y= \pm d / 2$ following a procedure presented in [Calas et al. 2008]. We also investigate classical radiation conditions at infinity through the study of an auxiliary problem involving (2-5) and (2-9).

3A. Statement of the main problem. We want to find $u, \varphi$, and $\psi$ describing an SH wave propagating in the positive $x$ direction that satisfy (2-1)-(2-3) in each phase of the heterogeneous structure A/B/A. Since we are interested in the study of confined modes, the functions $u, \varphi$, and $\psi$ in media A vanish when $y \rightarrow \pm \infty$ (evanescent waves in the $y$ direction). In [Calas et al. 2008] the dispersion relation for the case of perfect contact conditions at the interfaces was studied. Here, two types of imperfect contact conditions will be considered:

(a) Case of electromagnetically permeable bonding at $y= \pm d / 2$, for which

$$
T_{A}=T_{B}=K\left(u_{A}-u_{B}\right), \quad \varphi_{A}=\varphi_{B}, \quad D_{A}=D_{B}, \quad \psi_{A}=\psi_{B}, \quad B_{A}=B_{B} .
$$

(b) Case of electromagnetically absorbent bonding at $y= \pm d / 2$, for which

$$
T_{A}=T_{B}=K\left(u_{A}-u_{B}\right), \quad \varphi_{A}=0, \quad \varphi_{B}=0, \quad \psi_{A}=0, \quad \psi_{B}=0 .
$$

The variables $u, \varphi$, and $\psi$ were introduced before (2-1)-(2-3) and $T, D$, and $B$ were introduced before (2-4). Also, the subscripts $A$ and $B$ identify the MEE media A and B, respectively. The first conditions in (3-1) and (3-2) describe an elastic interface with spring constant material parameter $K>0$, which has dimensions of stress divided by length; in particular, $[K]=\mathrm{GPa} / \mathrm{m}$. We shall call this constant the interface parameter. With this model, the interface is allowed to deform and the displacement at the interface can be discontinuous. The case $K \rightarrow \infty$ corresponds to a perfectly bonded interface and the case $K \rightarrow 0$ corresponds to a mechanically free interface.

3B. The auxiliary problem. We now consider harmonic SH waves propagating in the positive $x$ direction and want to find solutions $u, \tilde{\varphi}$, and $\tilde{\psi}$ of (2-5) and (2-9) in regions A and B that satisfy the radiation conditions in regions $\mathrm{A}$, that is, functions $u, \tilde{\varphi}$, and $\tilde{\psi}$ vanish for $y= \pm \infty$. Using the symmetry properties of the heterostructure, as in [Calas et al. 2008], the MEE fields can be decoupled into symmetric and antisymmetric modes in $y$. Indeed, the linear equations (2-5) and (2-9) are invariant under reflections with respect to the $x$-axis in both regions $\mathrm{A}$ and $\mathrm{B}$. Consequently, the symmetric and antisymmetric parts with respect to $y$ of the solutions are also solutions of (2-5) and (2-9). In addition, it follows from the linear relations (2-7) and (2-12) that the fields $\varphi, \psi, T, D$, and $B$ are sums of the symmetric and antisymmetric parts of $u, \tilde{\varphi}$, and $\tilde{\psi}$. Consequently, conditions (3-1) and (3-2) at $y= \pm d / 2$ are valid for the symmetric and antisymmetric parts of the solutions.

From (2-5) and (2-9), and taking into account the above considerations, the following solutions are obtained:

$$
u=e^{i(\xi x-\omega t)}\left\{\begin{array}{lr}
U_{A} e^{\eta_{A} y}, & y \leq-d / 2 \\
U_{B} \cos \left(\eta_{B} y\right), & |y| \leq d / 2 \\
U_{A} e^{-\eta_{A} y}, & y \geq d / 2
\end{array}\right.
$$




$$
\begin{gathered}
\tilde{\varphi}=e^{i(\xi x-\omega t)}\left\{\begin{array}{lc}
\Phi_{A} e^{\xi y}, & y \leq-d / 2, \\
\Phi_{B} \cosh (\xi y), & |y| \leq d / 2, \\
\Phi_{A} e^{-\xi y}, & y \geq d / 2,
\end{array}\right. \\
\tilde{\psi}=e^{i(\xi x-\omega t)}\left\{\begin{array}{lc}
\Psi_{A} e^{\xi y}, & y \leq-d / 2, \\
\Psi_{B} \cosh (\xi y), & |y| \leq d / 2, \\
\Psi_{A} e^{-\xi y}, & y \geq d / 2,
\end{array}\right.
\end{gathered}
$$

for the symmetric modes in $y$, and

$$
\begin{gathered}
u=e^{i(\xi x-\omega t)}\left\{\begin{array}{lr}
U_{A}^{\prime} e^{\eta_{A} y}, & y \leq-d / 2, \\
U_{B}^{\prime} \sin \left(\eta_{B} y\right), & |y| \leq d / 2, \\
-U_{A}^{\prime} e^{-\eta_{A} y}, & y \geq d / 2,
\end{array}\right. \\
\tilde{\varphi}=e^{i(\xi x-\omega t)}\left\{\begin{array}{lc}
\Phi_{A}^{\prime} e^{\xi y}, & y \leq-d / 2, \\
\Phi_{B}^{\prime} \sinh (\xi y), & |y| \leq d / 2, \\
-\Phi_{A}^{\prime} e^{-\xi y}, & y \geq d / 2,
\end{array}\right. \\
\tilde{\psi}=e^{i(\xi x-\omega t)}\left\{\begin{array}{lc}
\Psi_{A}^{\prime} e^{\xi y}, & y \leq-d / 2, \\
\Psi_{B}^{\prime} \sinh (\xi y), & |y| \leq d / 2, \\
-\Psi_{A}^{\prime} e^{-\xi y}, & y \geq d / 2,
\end{array}\right.
\end{gathered}
$$

for the antisymmetric modes in $y$. Here, $\omega, \xi$, and $\eta$ are, respectively, the frequency, the $x$-component of the wave vector, and the $y$-component of the wave vector, whereas $U_{A}, U_{B}, \Phi_{A}, \Phi_{B}, \Psi_{A}$, and $\Psi_{B}$ are the amplitudes of the fields in the medium A and B. The parameters $\eta_{A}>0, \eta_{B}, \xi>0$, and $\omega$ appearing in (3-3)-(3-8) are related to each other through the expressions

$$
\begin{array}{ll}
\left(\eta_{A}\right)^{2}=(\xi)^{2}-\left(\omega / \bar{v}_{A}\right)^{2}=\xi^{2}\left(1-v^{2} / \bar{v}_{A}^{2}\right)>0, & |y| \geq d / 2, \\
\left(\eta_{B}\right)^{2}=\left(\omega / \bar{v}_{B}\right)^{2}-(\xi)^{2}=\xi^{2}\left(v^{2} / \bar{v}_{B}^{2}-1\right), & |y| \leq d / 2,
\end{array}
$$

where $v=\omega / \xi$ is the phase velocity.

Substituting (3-3)-(3-5) into (2-7), the symmetric parts of the electric and magnetic potentials are given by, respectively,

$$
\begin{aligned}
& \varphi=e^{i(\xi x-\omega t)} \begin{cases}\frac{\bar{e}_{A}}{\varepsilon_{A}} U_{A} e^{\eta_{A} y}+\chi_{A} \Phi_{A} e^{\xi y}-\frac{\bar{g}_{A}}{\varepsilon_{A}} \Psi_{A} e^{\xi y}, & y \leq-d / 2, \\
\frac{\bar{e}_{B}}{\varepsilon_{B}} U_{B} \cos \left(\eta_{B} y\right)+\chi_{B} \Phi_{B} \cosh (\xi y)-\frac{\bar{g}_{B}}{\varepsilon_{B}} \Psi_{B} \cosh (\xi y), & |y| \leq d / 2, \\
\frac{\bar{e}_{A}}{\varepsilon_{A}} U_{A} e^{-\eta_{A} y}+\chi_{A} \Phi_{A} e^{-\xi y}-\frac{\bar{g}_{A}}{\varepsilon_{A}} \Psi_{A} e^{-\xi y}, & y \geq d / 2,\end{cases} \\
& \psi=e^{i(\xi x-\omega t)}\left\{\begin{array}{lr}
\frac{\bar{f}_{A}}{\mu_{A}} U_{A} e^{\eta_{A} y}-\frac{\bar{g}_{A}}{\mu_{A}} \Phi_{A} e^{\xi y}+\chi_{A} \Psi_{A} e^{\xi y}, & y \leq-d / 2, \\
\frac{\bar{f}_{B}}{\mu_{B}} U_{B} \cos \left(\eta_{B} y\right)-\frac{\bar{g}_{B}}{\mu_{B}} \Phi_{B} \cosh (\xi y)+\chi_{B} \Psi_{B} \cosh (\xi y), & |y| \leq d / 2, \\
\frac{\bar{f}_{A}}{\mu_{A}} U_{A} e^{-\eta_{A} y}-\frac{\bar{g}_{A}}{\mu_{A}} \Phi_{A} e^{-\xi y}+\chi_{A} \Psi_{A} e^{-\xi y}, & y \geq d / 2 .
\end{array}\right.
\end{aligned}
$$


Substituting (3-3)-(3-5) into (2-12), we obtain the following expressions for the symmetric parts of the mechanical stress, electric displacement, and magnetic flux:

$$
\begin{aligned}
& T=e^{i(\xi x-\omega t)}\left\{\begin{array}{lr}
U_{A} \bar{c}_{A} \eta_{A} e^{\eta_{A} y}+\left(\bar{e}_{A} \Phi_{A}+\bar{f}_{A} \Psi_{A}\right) \xi e^{\xi y}, & y \leq-d / 2, \\
-U_{B} \bar{c}_{B} \eta_{B} \sin \left(\eta_{B} y\right)+\left(\bar{e}_{B} \Phi_{B}+\bar{f}_{B} \Psi_{B}\right) \xi \sinh (\xi y), & |y| \leq d / 2, \\
-U_{A} \bar{c}_{A} \eta_{A} e^{-\eta_{A} y}-\left(\bar{e}_{A} \Phi_{A}+\bar{f}_{A} \Psi_{A}\right) \xi e^{-\xi y}, & y \geq d / 2
\end{array}\right. \\
& D=e^{i(\xi x-\omega t)}\left\{\begin{array}{lc}
-\varepsilon_{A} \xi \Phi_{A} e^{\xi y}, & y \leq-d / 2, \\
-\varepsilon_{B} \xi \Phi_{B} \sinh (\xi y), & |y| \leq d / 2, \\
\varepsilon_{A} \xi \Phi_{A} e^{-\xi y}, & y \geq d / 2,
\end{array}\right. \\
& B=e^{i(\xi x-\omega t)}\left\{\begin{array}{lc}
-\mu_{A} \xi \Psi_{A} e^{\xi y}, & y \leq-d / 2, \\
-\mu_{B} \xi \Psi_{B} \sinh (\xi y), & |y| \leq d / 2, \\
\mu_{A} \xi \Psi_{A} e^{-\xi y}, & y \geq d / 2 .
\end{array}\right.
\end{aligned}
$$

Analogously, substituting (3-6)-(3-8) into (2-7) and (2-12), the antisymmetric parts of the electric and magnetic potentials, mechanical stress, electric displacement, and magnetic flux are given by

$$
\begin{aligned}
& \varphi=e^{i(\xi x-\omega t)} \begin{cases}\frac{\bar{e}_{A}}{\varepsilon_{A}} U_{A}^{\prime} e^{\eta_{A} y}+\chi_{A} \Phi_{A}^{\prime} e^{\xi y}-\frac{\bar{g}_{A}}{\varepsilon_{A}} \Psi_{A}^{\prime} e^{\xi y}, & y \leq-d / 2, \\
\frac{\bar{e}_{B}}{\varepsilon_{B}} U_{B}^{\prime} \sin \left(\eta_{B} y\right)+\chi_{B} \Phi_{B}^{\prime} \sinh (\xi y)-\frac{\bar{g}_{B}}{\varepsilon_{B}} \Psi_{B}^{\prime} \sinh (\xi y), & |y| \leq d / 2, \\
-\frac{\bar{e}_{A}}{\varepsilon_{A}} U_{A}^{\prime} e^{-\eta_{A} y}-\chi_{A} \Phi_{A}^{\prime} e^{-\xi y}+\frac{\bar{g}_{A}}{\varepsilon_{A}} \Psi_{A}^{\prime} e^{-\xi y}, & y \geq d / 2,\end{cases} \\
& \int \frac{\bar{f}_{A}}{\mu_{A}} U_{A}^{\prime} e^{\eta_{A} y}-\frac{\bar{g}_{A}}{\mu_{A}} \Phi_{A}^{\prime} e^{\xi y}+\chi_{A} \Psi_{A}^{\prime} e^{\xi y}, \quad y \leq-d / 2,
\end{aligned}
$$

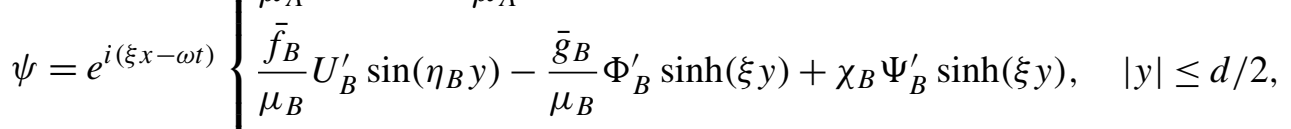

$$
\begin{aligned}
& -\frac{\bar{f}_{A}}{\mu_{A}} U_{A}^{\prime} e^{-\eta_{A} y}+\frac{\bar{g}_{A}}{\mu_{A}} \Phi_{A}^{\prime} e^{-\xi y}-\chi_{A} \Psi_{A}^{\prime} e^{-\xi y}, \\
& T=e^{i(\xi x-\omega t)}\left\{\begin{array}{lr}
U_{A}^{\prime} \bar{c}_{A} \eta_{A} e^{\eta_{A} y}+\left(\bar{e}_{A} \Phi_{A}^{\prime}+\bar{f}_{A} \Psi_{A}^{\prime}\right) \xi e^{\xi y}, & y \leq-d / 2, \\
U_{B}^{\prime} \bar{c}_{B} \eta_{B} \cos \left(\eta_{B} y\right)+\left(\bar{e}_{B} \Phi_{B}^{\prime}+\bar{f}_{B} \Psi_{B}^{\prime}\right) \xi \cosh (\xi y), & |y| \leq d / 2, \\
U_{A}^{\prime} \bar{c}_{A} \eta_{A} e^{-\eta_{A} y}+\left(\bar{e}_{A} \Phi_{A}^{\prime}+\bar{f}_{A} \Psi_{A}^{\prime}\right) \xi e^{-\xi y}, & y \geq d / 2,
\end{array}\right. \\
& D=e^{i(\xi x-\omega t)}\left\{\begin{array}{lc}
-\varepsilon_{A} \xi \Phi_{A}^{\prime} e^{\xi y}, & y \leq-d / 2, \\
-\varepsilon_{B} \xi \Phi_{B}^{\prime} \cosh (\xi y), & |y| \leq d / 2, \\
-\varepsilon_{A} \xi \Phi_{A}^{\prime} e^{-\xi y}, & y \geq d / 2
\end{array}\right. \\
& B=e^{i(\xi x-\omega t)}\left\{\begin{array}{lc}
-\mu_{A} \xi \Psi_{A}^{\prime} e^{\xi y}, & y \leq-d / 2, \\
-\mu_{B} \xi \Psi_{B}^{\prime} \cosh (\xi y), & |y| \leq d / 2, \\
-\mu_{A} \xi \Psi_{A}^{\prime} e^{-\xi y}, & y \geq d / 2 .
\end{array}\right.
\end{aligned}
$$




\section{Dispersion relations for imperfect contacts}

Now, we refer to the conditions at the interface between the layers, which is located at $y= \pm d / 2$, and the semiinfinite media. Due to the symmetry of the problem, such conditions are restricted to only one interface, and we study the dispersion relation for the imperfect bonding effects defined by (3-1) and (3-2) for the symmetric and antisymmetric solutions.

\section{A. Dispersion relation for electromagnetically permeable bonding.}

Symmetric modes. Substituting (3-3) and (3-10)-(3-14) into the interface conditions given by (3-1) for $y=-d / 2$, an homogeneous system of linear algebraic equations for $U_{A}, U_{B}, \Phi_{A}, \Phi_{B}, \Psi_{A}$, and $\Psi_{B}$ is obtained. For nontrivial solutions, the determinant of the principal matrix has to be zero, which leads to the following dispersion relation for the symmetric modes:

$$
P_{A} P_{B}-Q^{2}+K\left(P_{A}+P_{B}+2 Q\right)=0,
$$

where

$$
\begin{aligned}
P_{A} & =-\bar{c}_{A}\left(\eta_{A}-\bar{\xi}_{A A}^{2} \xi \sinh (\xi d / 2)\right), \\
P_{B} & =\bar{c}_{B}\left(\eta_{B} \tan \left(\eta_{B} d / 2\right)+\bar{\xi}_{B B}^{2} \xi \sinh (\xi d / 2)\right), \\
Q & =-\sqrt{\bar{c}_{A} \bar{c}_{B}} \bar{\xi}_{A B}^{2} \xi \sinh (\xi d / 2), \\
\bar{\xi}_{\alpha \beta}^{2} & =\frac{1}{\sqrt{\bar{c}_{\alpha} \bar{c}_{\beta}}} \frac{\frac{\bar{e}_{\alpha} \bar{e}_{\beta}}{\varepsilon_{\alpha} \varepsilon_{\beta}} \Upsilon_{A B}^{(1)}+\frac{\bar{f}_{\alpha} \bar{f}_{\beta}}{\mu_{\alpha} \mu_{\beta}} \Upsilon_{A B}^{(2)}+\left(\frac{\bar{e}_{\alpha} \bar{f}_{\beta}}{\varepsilon_{\alpha} \mu_{\beta}}+\frac{\bar{e}_{\beta} \bar{f}_{\alpha}}{\varepsilon_{\beta} \mu_{\alpha}}\right) \Upsilon_{A B}^{(3)}}{\Upsilon_{A B}^{(1)} \Upsilon_{A B}^{(2)}-\left(\Upsilon_{A B}^{(3)}\right)^{2}}, \\
\Upsilon_{A B}^{(1)} & =\frac{\chi_{A}}{\mu_{A}} \sinh (\xi d / 2)+\frac{\chi_{B}}{\mu_{B}} \cosh (\xi d / 2), \quad \Upsilon_{A B}^{(2)}=\frac{\chi_{A}}{\varepsilon_{A}} \sinh (\xi d / 2)+\frac{\chi_{B}}{\varepsilon_{B}} \cosh (\xi d / 2), \\
\Upsilon_{A B}^{(3)} & =\frac{\bar{g}_{A}}{\varepsilon_{A} \mu_{A}} \sinh (\xi d / 2)+\frac{\bar{g}_{B}}{\varepsilon_{B} \mu_{B}} \cosh (\xi d / 2) .
\end{aligned}
$$

Here, $\bar{\xi}_{\alpha \beta}$, with $\alpha \beta=A A, B B, A B$, is a coupling constant, which depends on the MEE properties of the phases and the thickness $d$ of the layer.

Let us consider the following limit cases:

(i) Materials A and B are piezoelectric. Expressions (4-2)-(4-4) reduce to

$$
\begin{gathered}
P_{A}=-\bar{c}_{A}^{e}\left(\eta_{A}-\frac{e_{A}^{2} \xi \bar{\varepsilon}}{\bar{c}_{A}^{e} \varepsilon_{A}(\bar{\varepsilon}+\operatorname{coth}(\xi d / 2))}\right), \\
P_{B}=\bar{c}_{B}^{e}\left(\eta_{B} \tan \left(\eta_{B} d / 2\right)+\frac{e_{B}^{2} \xi}{\bar{c}_{B}^{e} \varepsilon_{B}(\bar{\varepsilon}+\operatorname{coth}(\xi d / 2))}\right), \\
Q=-\frac{e_{A} e_{B} \xi}{\varepsilon_{A}(\bar{\varepsilon}+\operatorname{coth}(\xi d / 2))},
\end{gathered}
$$

where $\bar{c}_{\alpha}^{e}=c_{\alpha}+e_{\alpha}^{2} / \varepsilon_{\alpha}$ is the piezoelectrically stiffened elastic constant and $\bar{\varepsilon}=\varepsilon_{B} / \varepsilon_{A}$. 
(ii) Materials A and B are piezomagnetic. Expressions (4-2)-(4-4) reduce to

$$
\begin{aligned}
P_{A} & =-\bar{c}_{A}^{f}\left(\eta_{A}-\frac{f_{A}^{2} \xi \bar{\mu}}{\bar{c}_{A}^{f} \mu_{A}(\bar{\mu}+\operatorname{coth}(\xi d / 2))}\right), \\
P_{B} & =\bar{c}_{B}^{f}\left(\eta_{B} \tan \left(\eta_{B} d / 2\right)+\frac{f_{B}^{2} \xi}{\bar{c}_{B}^{f} \mu_{B}(\bar{\mu}+\operatorname{coth}(\xi d / 2))}\right), \\
Q & =-\frac{f_{A} f_{B} \xi}{\mu_{A}(\bar{\mu}+\operatorname{coth}(\xi d / 2))},
\end{aligned}
$$

where $\bar{c}_{\alpha}^{f}=c_{\alpha}+f_{\alpha}^{2} / \mu_{\alpha}$ is the piezomagnetically stiffened elastic constant and $\bar{\mu}=\mu_{B} / \mu_{A}$.

(iii) Material A is piezoelectric and material B is piezomagnetic. Expressions (4-2)-(4-4) reduce to (4-8), (4-12), and $Q=0$, respectively.

(iv) Material A is piezomagnetic and material B is piezoelectric. Expressions (4-2)-(4-4) reduce to (4-11), (4-9), and $Q=0$, respectively.

(v) The interface is perfectly bonded. Expression (4-1) reduces to

$$
P_{A}+P_{B}+2 Q=0
$$

Substituting (4-2)-(4-7) into (4-14), we obtain the dispersion relation [Calas et al. 2008, Equation (59)].

(vi) The interface has no mechanical interaction, $K=0$, and the media can interact magnetoelectrically. In this case, (4-1) can be written as

$$
P_{A} P_{B}-Q^{2}=0
$$

Substituting (4-2)-(4-7) into (4-15), we obtain

$$
\left(\eta_{A}-\bar{\xi}_{A A}^{2} \xi \sinh (\xi d / 2)\right)\left(\eta_{B} \tan \left(\eta_{B} d / 2\right)+\bar{\xi}_{B B}^{2} \xi \sinh (\xi d / 2)\right)=-\left(\bar{\xi}_{A B}^{2} \xi \sinh (\xi d / 2)\right)^{2},
$$

which, together with both (3-9) and $v=\omega / \xi$, yields

$$
\begin{aligned}
-\left(\bar{\xi}_{A B}^{2} \sinh \left(\frac{1}{v} \frac{\omega d}{2}\right)\right)^{2}=((1 & \left.\left.-\frac{v^{2}}{\bar{v}_{A}^{2}}\right)^{\frac{1}{2}}-\bar{\xi}_{A A}^{2} \sinh \left(\frac{1}{v} \frac{\omega d}{2}\right)\right) \\
& \times\left(\left(\frac{v^{2}}{\bar{v}_{B}^{2}}-1\right)^{\frac{1}{2}} \tan \left(\left(\frac{v^{2}}{\bar{v}_{B}^{2}}-1\right)^{\frac{1}{2}} \frac{1}{v} \frac{\omega d}{2}\right)+\bar{\xi}_{B B}^{2} \sinh \left(\frac{1}{v} \frac{\omega d}{2}\right)\right) .
\end{aligned}
$$

Note from (4-17) that if $\omega d=0$, then $v$ is given by

$$
v=\bar{v}_{A}
$$

which is the bulk shear wave of medium A. 
Antisymmetric modes. Following a procedure similar to the one used for symmetric modes, we substitute (3-6) and (3-15)-(3-19) into the interface conditions of Case (a) (page (3-1)) at $y=-d / 2$, and obtain the wave speed equation for the antisymmetric modes in the form of Equation (4-1), where

$$
\begin{aligned}
P_{A} & =-\bar{c}_{A}\left(\eta_{A}-\bar{\xi}_{A A}^{2} \xi \cosh (\xi d / 2)\right), \\
P_{B} & =-\bar{c}_{B}\left(\eta_{B} \cot \left(\eta_{B} d / 2\right)-\bar{\xi}_{B B}^{2} \xi \cosh (\xi d / 2)\right), \\
Q & =-\sqrt{\bar{c}_{A} \bar{c}_{B}} \bar{\xi}_{A B}^{2} \xi \cosh (\xi d / 2),
\end{aligned}
$$

and $\bar{\xi}_{\alpha \beta}$ can be calculated using (4-5), but with $\Upsilon_{A B}^{(i)}(i=1,2,3)$ given by

$$
\begin{gathered}
\Upsilon_{A B}^{(1)}=\frac{\chi_{A}}{\mu_{A}} \cosh (\xi d / 2)+\frac{\chi_{B}}{\mu_{B}} \sinh (\xi d / 2), \quad \Upsilon_{A B}^{(2)}=\frac{\chi_{A}}{\varepsilon_{A}} \cosh (\xi d / 2)+\frac{\chi_{B}}{\varepsilon_{B}} \sinh (\xi d / 2), \\
\Upsilon_{A B}^{(3)}=\frac{\bar{g}_{A}}{\varepsilon_{A} \mu_{A}} \cosh (\xi d / 2)+\frac{\bar{g}_{B}}{\varepsilon_{B} \mu_{B}} \sinh (\xi d / 2) .
\end{gathered}
$$

Let us consider the following limit cases:

(i) Materials A and B are piezoelectric. Expressions (4-19)-(4-21) reduce to

$$
\begin{aligned}
P_{A} & =-\bar{c}_{A}^{e}\left(\eta_{A}-\frac{e_{A}^{2} \xi \bar{\varepsilon}}{\bar{c}_{A}^{e} \varepsilon_{A}(\bar{\varepsilon}+\tanh (\xi d / 2))}\right), \\
P_{B} & =-\bar{c}_{B}^{e}\left(\eta_{B} \cot \left(\eta_{B} d / 2\right)-\frac{e_{B}^{2} \xi}{\bar{c}_{B}^{e} \varepsilon_{B}(\bar{\varepsilon}+\tanh (\xi d / 2))}\right), \\
Q & =-\frac{e_{A} e_{B} \xi}{\varepsilon_{A}(\bar{\varepsilon}+\tanh (\xi d / 2))} .
\end{aligned}
$$

(ii) Materials A and B are piezomagnetic. Expressions (4-19)-(4-21) reduce to

$$
\begin{aligned}
P_{A} & =-\bar{c}_{A}^{f}\left(\eta_{A}-\frac{f_{A}^{2} \xi \bar{\mu}}{\bar{c}_{A}^{f} \mu_{A}(\bar{\mu}+\tanh (\xi d / 2))}\right), \\
P_{B} & =-\bar{c}_{B}^{f}\left(\eta_{B} \cot \left(\eta_{B} d / 2\right)-\frac{f_{B}^{2} \xi}{\bar{c}_{B}^{f} \mu_{B}(\bar{\mu}+\tanh (\xi d / 2))}\right), \\
Q & =-\frac{f_{A} f_{B} \xi}{\mu_{A}(\bar{\mu}+\tanh (\xi d / 2))} .
\end{aligned}
$$

(iii) Material A is piezoelectric and material B is piezomagnetic. Expressions (4-19)-(4-21) reduce to (4-24), (4-28), and $Q=0$, respectively.

(iv) Material A is piezomagnetic and material B is piezoelectric. Expressions (4-19)-(4-21) reduce to (4-27), (4-25), and $Q=0$, respectively.

(v) The interface is perfectly bonded. Expression (4-1) reduces to (4-14).

(vi) The interface has no mechanical interaction, $K=0$, and the media can interact magnetoelectrically. Equation (4-1) can be written as (4-15). Substituting (4-19)-(4-23) into (4-15), we obtain

$$
\left(\eta_{A}-\bar{\xi}_{A A}^{2} \xi \cosh (\xi d / 2)\right)\left(\eta_{B} \cot \left(\eta_{B} d / 2\right)-\bar{\xi}_{B B}^{2} \xi \cosh (\xi d / 2)\right)=\left(\bar{\xi}_{A B}^{2} \xi \cosh (\xi d / 2)\right)^{2},
$$


which, together with both (3-9) and $v=\omega / \xi$, yields

$$
\begin{aligned}
\left(\bar{\xi}_{A B}^{2} \cosh \left(\frac{1}{v} \frac{\omega d}{2}\right)\right)^{2}=\left(\left(1-\frac{v^{2}}{v_{A}^{2}}\right)^{\frac{1}{2}}-\bar{\xi}_{A A}^{2} \cosh \left(\frac{1}{v} \frac{\omega d}{2}\right)\right) \\
\quad \times\left(\left(\frac{v^{2}}{v_{B}^{2}}-1\right)^{\frac{1}{2}} \cot \left(\left(\frac{v^{2}}{v_{B}^{2}}-1\right)^{\frac{1}{2}} \frac{1}{v} \frac{\omega d}{2}\right)-\bar{\xi}_{B B}^{2} \cosh \left(\frac{1}{v} \frac{\omega d}{2}\right)\right) .
\end{aligned}
$$

Equation (4-31) governs the magnetoelectrical interaction between the three phases. Note from (4-31) that if $\omega d=0,(4-31)$ yields the following velocity on the half-spaces $(|y|>d / 2)$ :

$$
v^{2}=\bar{v}_{A}^{2}\left(1-\bar{\xi}_{A}^{4}\right)
$$

where

$$
\bar{\xi}_{A}^{2}=1-\frac{c_{A}}{\bar{c}_{A}}
$$

is the limit of $\bar{\xi}_{A A}$ when $\omega d \rightarrow 0$. The magnitude $v$ defined by (4-32) is the speed of the BleusteinGulyaev wave for medium A (see, [Bleustein 1968; Gulyaev 1969]). These results are in agreement with other similar studies, for instance, [Fan et al. 2006a, (15) and (16)] or [Melkumyan and Mai 2008, (27)].

\section{B. Dispersion relation for electromagnetically absorbent bonding.}

Symmetric modes. Substituting (3-3) and (3-10)-(3-12) into the interface conditions given by (3-2) for $y=-d / 2$, we obtain the dispersion relation for the symmetric modes, which is given by

$$
P_{A} P_{B}+K\left(P_{A}+P_{B}\right)=0,
$$

where

$$
\begin{aligned}
P_{A} & =-\bar{c}_{A}\left(\eta_{A}-\bar{\xi}_{A}^{2} \xi\right), \\
P_{B} & =\bar{c}_{B}\left(\eta_{B} \tan \left(\eta_{B} d / 2\right)+\bar{\xi}_{B}^{2} \xi \tanh (\xi d / 2)\right), \\
\bar{\xi}_{\alpha}^{2} & =1-\frac{c_{\alpha}}{\bar{c}_{\alpha}},
\end{aligned}
$$

with $\bar{\xi}_{\alpha}$, for $\alpha=A, B$, being a coupling constant of the MEE homogeneous medium $\alpha$. From (4-37) and using (2-11) on each phase, one can obtain [Huang and Li 2010, (18)].

Let us consider the following special cases:

(i) Materials A and B are piezoelectric. Expressions (4-35) and (4-36) reduce to

$$
\begin{aligned}
& P_{A}=-\bar{c}_{A}^{e}\left(\eta_{A}-\frac{e_{A}^{2} \xi}{\bar{c}_{A}^{e} \varepsilon_{A}}\right), \\
& P_{B}=\bar{c}_{B}^{e}\left(\eta_{B} \tan \left(\eta_{B} d / 2\right)+\frac{e_{B}^{2} \xi}{\bar{c}_{B}^{e} \varepsilon_{B}} \tanh (\xi d / 2)\right) .
\end{aligned}
$$

(ii) Materials A and B are piezomagnetic. Expressions (4-35) and (4-36) reduce to

$$
\begin{aligned}
& P_{A}=-\bar{c}_{A}^{f}\left(\eta_{A}-\frac{f_{A}^{2} \xi}{\bar{c}_{A}^{f} \mu_{A}}\right), \\
& P_{B}=\bar{c}_{B}^{f}\left(\eta_{B} \tan \left(\eta_{B} d / 2\right)+\frac{f_{B}^{2} \xi}{\bar{c}_{B}^{f} \mu_{B}} \tanh (\xi d / 2)\right) .
\end{aligned}
$$


(iii) Material A is piezoelectric and material B is piezomagnetic. Expressions (4-35) and (4-36) reduce to (4-38) and (4-41), respectively.

(iv) Material A is piezomagnetic and material B is piezoelectric. Expressions (4-35) and (4-36) reduce to (4-40) and (4-39), respectively.

(v) The interface is perfectly bonded. Expression (4-34) reduces to

$$
-\bar{c}_{A}\left(\eta_{A}-\bar{\xi}_{A}^{2} \xi\right)+\bar{c}_{B}\left(\eta_{B} \tan \left(\eta_{B} d / 2\right)+\bar{\xi}_{B}^{2} \xi \tanh (\xi d / 2)\right)=0 .
$$

Equation (4-42) can be written as a function of the normalized velocity $V_{\alpha}=v / \bar{v}_{\alpha}, \alpha=A, B$, and the dimensionless frequency $\Omega=\omega d /\left(\pi \bar{v}_{B}\right)$ as

$\bar{c}_{B}\left(\left(V_{B}^{2}-1\right)^{1 / 2} \tan \left(\frac{\pi}{2} \frac{\Omega}{V_{B}}\left(V_{B}^{2}-1\right)^{1 / 2}\right)+\bar{\xi}_{B}^{2} \tanh \left(\frac{\pi}{2} \frac{\Omega}{V_{B}}\right)\right)-\bar{c}_{A}\left(\left(1-V_{A}^{2}\right)^{1 / 2}-\bar{\xi}_{A}^{2}\right)=0$.

Setting $\Omega \rightarrow 0$, (4-43) reduces to the velocity of the Bleustein-Gulyaev wave given by (4-32) in the two half-spaces $y>d / 2$ and $y<-d / 2$. These results are in agreement with [Fan et al. 2006b, Equations (10) and (11)] and also [Huang et al. 2009, Equations (19) and (20)].

(vi) The interface has no mechanical-magnetoelectrical interaction. Equation (4-34) can be written as

$$
P_{A} P_{B}=0 \text {. }
$$

For $P_{A}=0$, the phase velocity is identical to (4-32), the velocity of the Bleustein-Gulyaev wave. On the other hand, if $P_{B}=0$, the velocity in the layer $|y|<d / 2$ satisfies the equation

$$
\eta_{B} \tan \left(\eta_{B} d / 2\right)+\bar{\xi}_{B}^{2} \xi \tanh (\xi d / 2)=0 .
$$

The roots of (4-45) determine the dispersion curves for a plate with grounded electrodes. It can be written, in a way similar to [Bleustein 1969, Equation (27)], as

$$
\frac{\tan \left(\frac{1}{2} \pi\left(\Omega^{2}-Z^{2}\right)^{1 / 2}\right)}{\tanh \left(\frac{1}{2} \pi Z\right)}=-\frac{\bar{\xi}_{B}^{2} Z}{\left(\Omega^{2}-Z^{2}\right)^{1 / 2}},
$$

where the dimensionless wave number $(Z)$ in the $x$ direction is defined by

$$
Z=\frac{\xi d}{\pi}
$$

In the limit when $Z \rightarrow 0$, (4-46) reduces to

$$
\tan \left(\frac{\pi}{2} \Omega\right)=0, \quad \omega d=n \pi \bar{v}_{B}, \quad n=0,2,4,6, \ldots,
$$

which is the frequency equation for the symmetric modes of a MEE plate. Equation (4-45) can also be written as a function of the normalized velocity $V_{B}$ and the dimensionless wave number $Z$ as

$$
\frac{\tan \left(\frac{1}{2} \pi Z\left(V_{B}^{2}-1\right)^{1 / 2}\right)}{\tanh \left(\frac{1}{2} \pi Z\right)}=-\frac{\bar{\xi}_{B}^{2}}{\left(V_{B}^{2}-1\right)^{1 / 2}}
$$

Taking $Z \rightarrow 0$, (4-49) reduces to

$$
V_{B}^{2}=\left(1-\bar{\xi}_{B}^{2}\right), \quad \text { or } \quad v^{2}=\bar{v}_{B}^{2}\left(1-\bar{\xi}_{B}^{2}\right) .
$$


Equation (4-45) can also be written as a function of the normalized velocity $V_{B}$ and the dimensionless frequency $\Omega$ as

$$
\frac{\tan \left(\frac{1}{2} \pi\left(\Omega / V_{B}\right)\left(V_{B}^{2}-1\right)^{1 / 2}\right)}{\tanh \left(\frac{1}{2} \pi\left(\Omega / V_{B}\right)\right)}=-\frac{\bar{\xi}_{B}^{2}}{\left(V_{B}^{2}-1\right)^{1 / 2}} .
$$

Note that (4-51) reduces to (4-50) for $\Omega \rightarrow 0$.

Antisymmetric modes. Substituting (3-6) and (3-15)-(3-17) into the conditions given by (3-2) for $y=$ $-d / 2$, the dispersion relation for the antisymmetric modes can be written as (4-34), with

$$
P_{B}=-\bar{c}_{B}\left(\eta_{B} \cot \left(\eta_{B} d / 2\right)-\bar{\xi}_{B}^{2} \xi \operatorname{coth}(\xi d / 2)\right),
$$

where $P_{A}$ and $\bar{\xi}_{\alpha}^{2}$ are given by (4-35) and (4-37), respectively.

Let us consider the following limit cases:

(i) Materials A and B are piezoelectric. Expression (4-35) becomes (4-38) and (4-52) takes the form

$$
P_{B}=-\bar{c}_{B}^{e}\left(\eta_{B} \cot \left(\eta_{B} d / 2\right)-\frac{e_{B}^{2} \xi}{\bar{c}_{B}^{e} \varepsilon_{B}} \operatorname{coth}(\xi d / 2)\right)
$$

(ii) Materials A and B are piezomagnetic. Expression (4-35) reduces to (4-40) and (4-52) becomes

$$
P_{B}=-\bar{c}_{B}^{f}\left(\eta_{B} \cot \left(\eta_{B} d / 2\right)-\frac{f_{B}^{2} \xi}{\bar{c}_{B}^{f} \mu_{B}} \operatorname{coth}(\xi d / 2)\right) .
$$

(iii) Material A is piezoelectric and material B is piezomagnetic. Expressions (4-35) and (4-52) reduce to (4-38) and (4-54), respectively.

(iv) Material A is piezomagnetic and material B is piezoelectric. Expressions (4-35) and (4-52) reduce to (4-40) and (4-53), respectively.

(v) The interface is perfectly bonded. Expression (4-34) reduces to

$$
-\bar{c}_{A}\left(\left(1-V_{A}^{2}\right)^{1 / 2}-\bar{\xi}_{A}^{2}\right)=\bar{c}_{B} \bar{\xi}_{B}^{2} \operatorname{coth}\left(\frac{\pi}{2} \frac{\Omega}{V_{B}}\right)+\bar{c}_{B}\left(\left(V_{B}^{2}-1\right)^{1 / 2} \cot \left(\frac{\pi}{2} \frac{\Omega}{V_{B}}\left(V_{B}^{2}-1\right)^{1 / 2}\right)\right) .
$$

Setting $\Omega \rightarrow 0$, (4-55) does not have a solution. In fact, dividing by $\operatorname{coth}\left(\frac{1}{2} \pi\left(\Omega / V_{B}\right)\right)$ and taking the limit for $\Omega \rightarrow 0$ results in $\bar{\xi}_{B}^{2}=1$, which is not possible.

(vi) The interface has no mechanical-magnetoelectrical interaction. From (4-34), the expression $P_{A} P_{B}=$ 0 is obtained. If $P_{A}=0$, the phase velocity is identical to (4-32), and if $P_{B}=0$, the phase velocity for the layer is given by an expression that is similar to [Bleustein 1969, Equation (23)] and is obtained from the dispersion equation

$$
\frac{\tan \left(\frac{1}{2} \pi\left(\Omega^{2}-Z^{2}\right)^{1 / 2}\right)}{\tanh \left(\frac{1}{2} \pi Z\right)}=\frac{\left(\Omega^{2}-Z^{2}\right)^{1 / 2}}{\bar{\xi}_{B}^{2} Z} .
$$

In the limit, as $Z \rightarrow 0$, (4-56) reduces to

$$
\tan \left(\frac{\pi}{2} \Omega\right)=\frac{\pi \Omega}{2 \bar{\xi}_{B}^{2}}
$$


which is the frequency equation for the antisymmetric modes of a MEE plate. Equation (4-56) can be written as a function of the normalized velocity $V_{B}$ and the dimensionless frequency $\Omega$ as

$$
\frac{\tan \left(\frac{1}{2} \pi\left(\Omega / V_{B}\right)\left(V_{B}^{2}-1\right)^{1 / 2}\right)}{\tanh \left(\frac{1}{2} \pi\left(\Omega / V_{B}\right)\right)}=\frac{\left(V_{B}^{2}-1\right)^{1 / 2}}{\bar{\xi}_{B}^{2}} .
$$

When $\Omega \rightarrow 0$, (4-58) reduces to $v=\bar{v}_{B}$.

\section{Numerical examples}

We now show dispersion curves for different heterostructures of type A/B/A with MEE properties and discuss quantitative and qualitative aspects of bonding on the dynamic properties of MEE heterostructures. An important aspect is that the behavior of the dispersion curves is strongly dependent on the spring constant material parameter $K$.

To perform numerical calculations, we use the piezomagnetic material $\mathrm{CoFe}_{2} \mathrm{O}_{4}$, the piezoelectric materials $\mathrm{BaTiO}_{3}$ and PZT4, and the composite $\mathrm{BaTiO}_{3} / \mathrm{CoFe}_{2} \mathrm{O}_{4}$ with 30 percent $\mathrm{BaTiO}_{3}$ [BravoCastillero et al. 2008]. This composite material exhibits MEE properties. We then analyze the dispersion of waves considering the first mode of vibration in the heterostructures $\mathrm{BaTiO}_{3} / \mathrm{CoFe}_{2} \mathrm{O}_{4} / \mathrm{BaTiO}_{3}$, $\mathrm{CoFe}_{2} \mathrm{O}_{4} / \mathrm{PZT} 4 / \mathrm{CoFe}_{2} \mathrm{O}_{4}$, and Composite/CoFe $\mathrm{O}_{4} /$ Composite with the bonding conditions (3-1), modeling a permeable interface, and (3-2), modeling an absorbent interface. The MEE properties used in the calculations are given in Table 1 . The dimensions of the interface parameter $K$, which we recall from Section $3 \mathrm{~A}$ are $\mathrm{GPa} / \mathrm{m}$, are omitted in Figures 2-8.

In Figure 2 we show dispersion curves for the symmetric parts of $\mathrm{SH}$ waves propagating in the heterostructure of $\mathrm{BaTiO}_{3} / \mathrm{CoFe}_{2} \mathrm{O}_{4} / \mathrm{BaTiO}_{3}$ with either a permeable (Figure 2a) or absorbent (Figure 2b) interface for increasing values of the spring constant $K$. Observe from Figure $2 \mathrm{a}$ that these curves are nonintersecting, nonmonotonic for small values of $K$, and both strictly decreasing and convex for large values of $K$. These last features can also be observed in [Calas et al. 2008, Figure 2]. In particular, all wave velocities tend to the wave velocity in phase $\mathrm{B}, \bar{v}_{\mathrm{CoFe}_{2} \mathrm{O}_{4}}$, as $\omega d \rightarrow \infty$. We conclude from this observation, which is also valid for Figure $2 b$, that the presence of imperfections at the interfaces does

\begin{tabular}{|llclc|}
\hline Properties & $\mathrm{BaTiO}_{3}$ & $\mathrm{CoFe}_{2} \mathrm{O}_{4}$ & PZT4 & Composite \\
$c(\mathrm{GPa})$ & 43 & 45.3 & 26 & 44.58 \\
$e\left(\mathrm{C} / \mathrm{m}^{2}\right)$ & 11.6 & 0 & 10.5 & 3.61 \\
$f(\mathrm{~N} / \mathrm{Am})$ & 0 & 550 & 0 & 378.92 \\
$g\left(10^{-9} \mathrm{Ns} / \mathrm{Vm}\right)$ & 0 & 0 & 0 & -30.67 \\
$\varepsilon\left(10^{-9} \mathrm{C}^{2} / \mathrm{Nm}^{2}\right)$ & 11.2 & 0.08 & 7.124 & 4.06 \\
$\mu\left(10^{-6} \mathrm{~N} \mathrm{~s} / \mathrm{C}^{2}\right)$ & 5 & 590 & 5 & 415.95 \\
$\rho\left(10^{3} \mathrm{Kg} / \mathrm{m}^{3}\right)$ & 5.8 & 5.3 & 7.5 & 5.45 \\
$\bar{v}\left(10^{3} \mathrm{~m} / \mathrm{s}\right)$ & 3.07981 & 2.94005 & 2.35162 & 2.97348 \\
$\bar{v}_{\mathrm{B}-\mathrm{G}}\left(10^{3} \mathrm{~m} / \mathrm{s}\right)$ & 3.00547 & 2.93987 & 2.18178 & 2.96514 \\
\hline
\end{tabular}

Table 1. Material properties. 


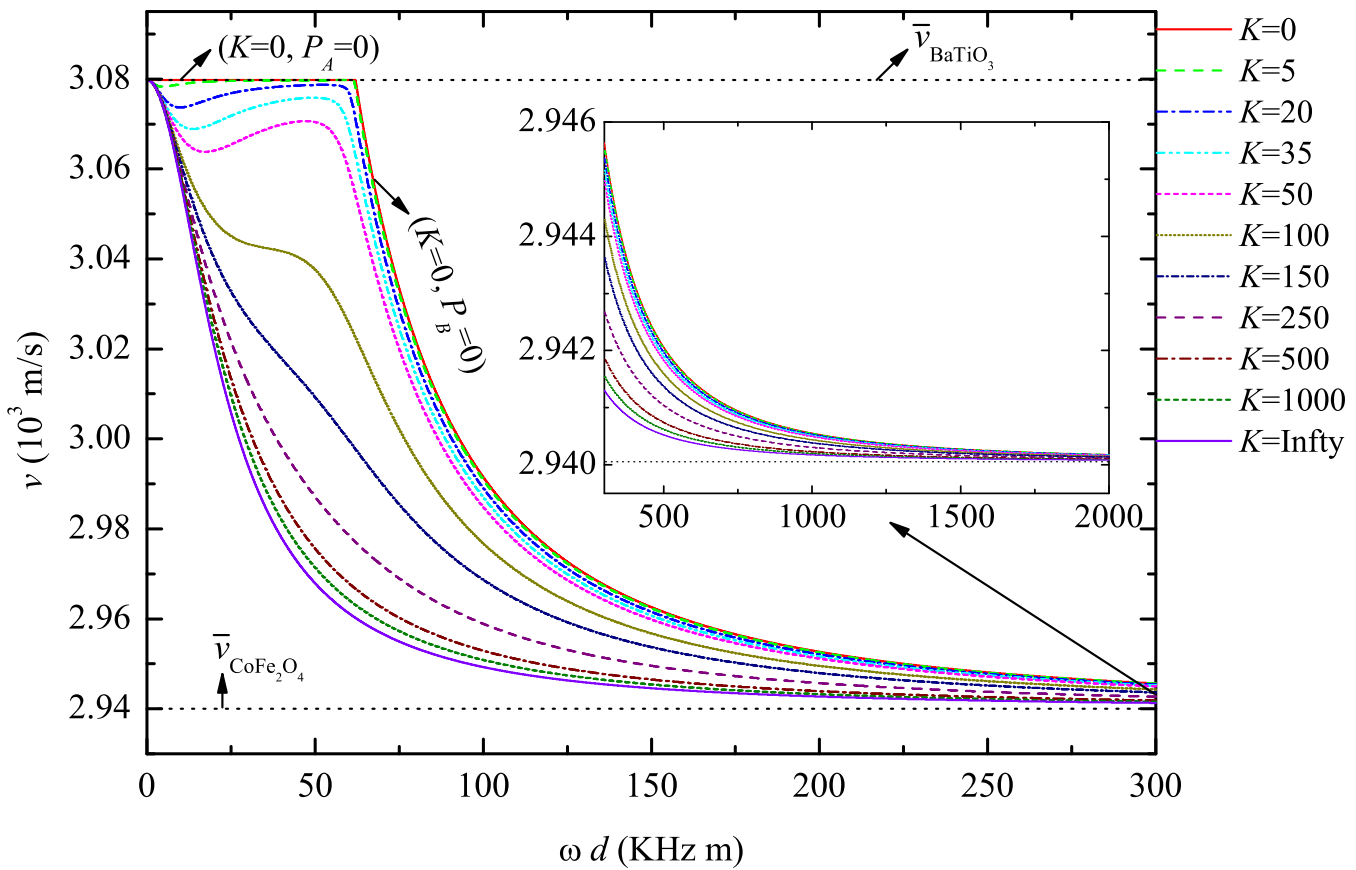

(a)

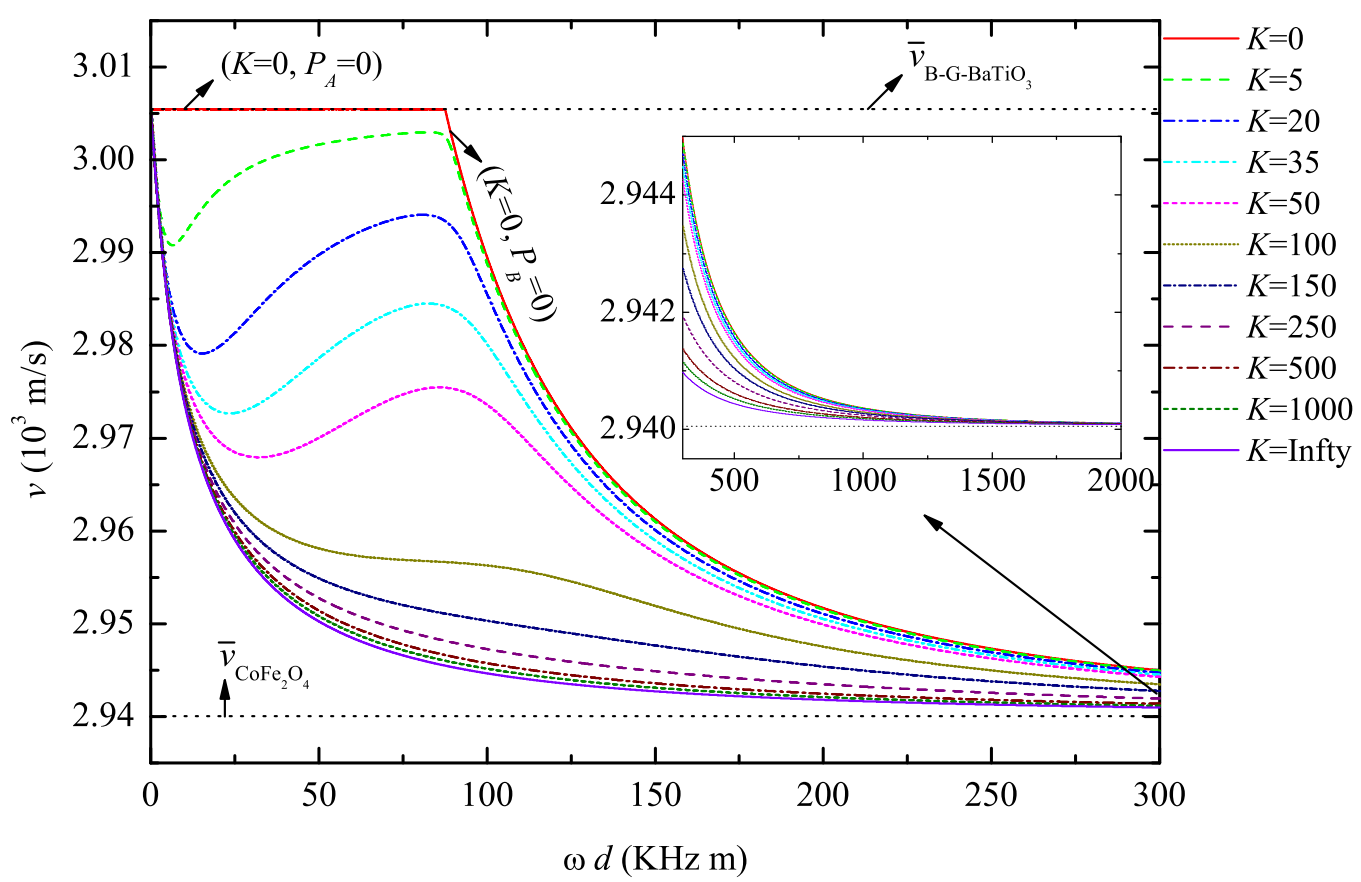

(b)

Figure 2. Influence of imperfect bonding on the dispersion curves for the symmetric modes in a $\mathrm{BaTiO}_{3} / \mathrm{CoFe}_{2} \mathrm{O}_{4} / \mathrm{BaTiO}_{3}$ heterostructure: (a) permeable and (b) absorbent bonding. 


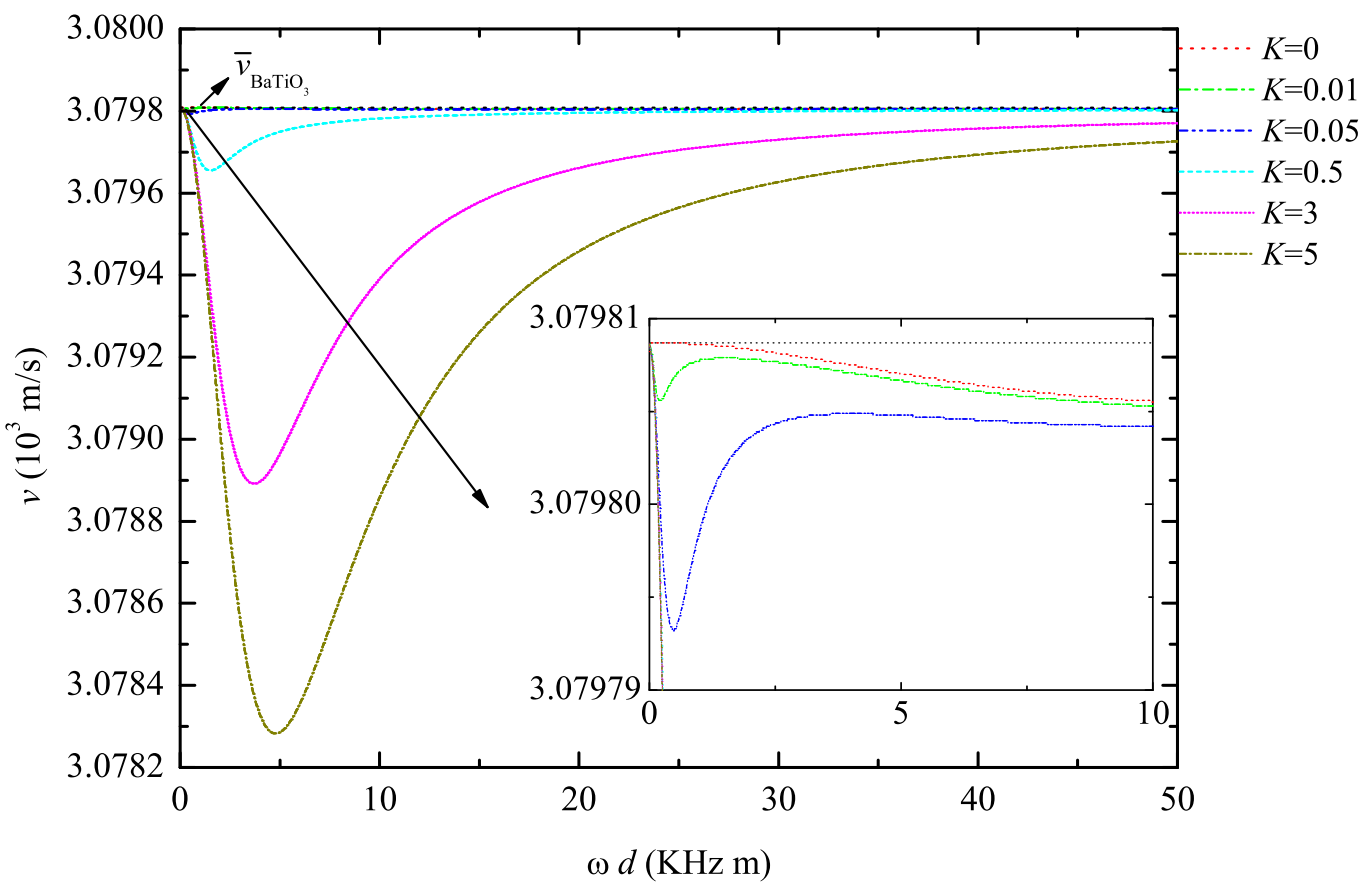

(a)

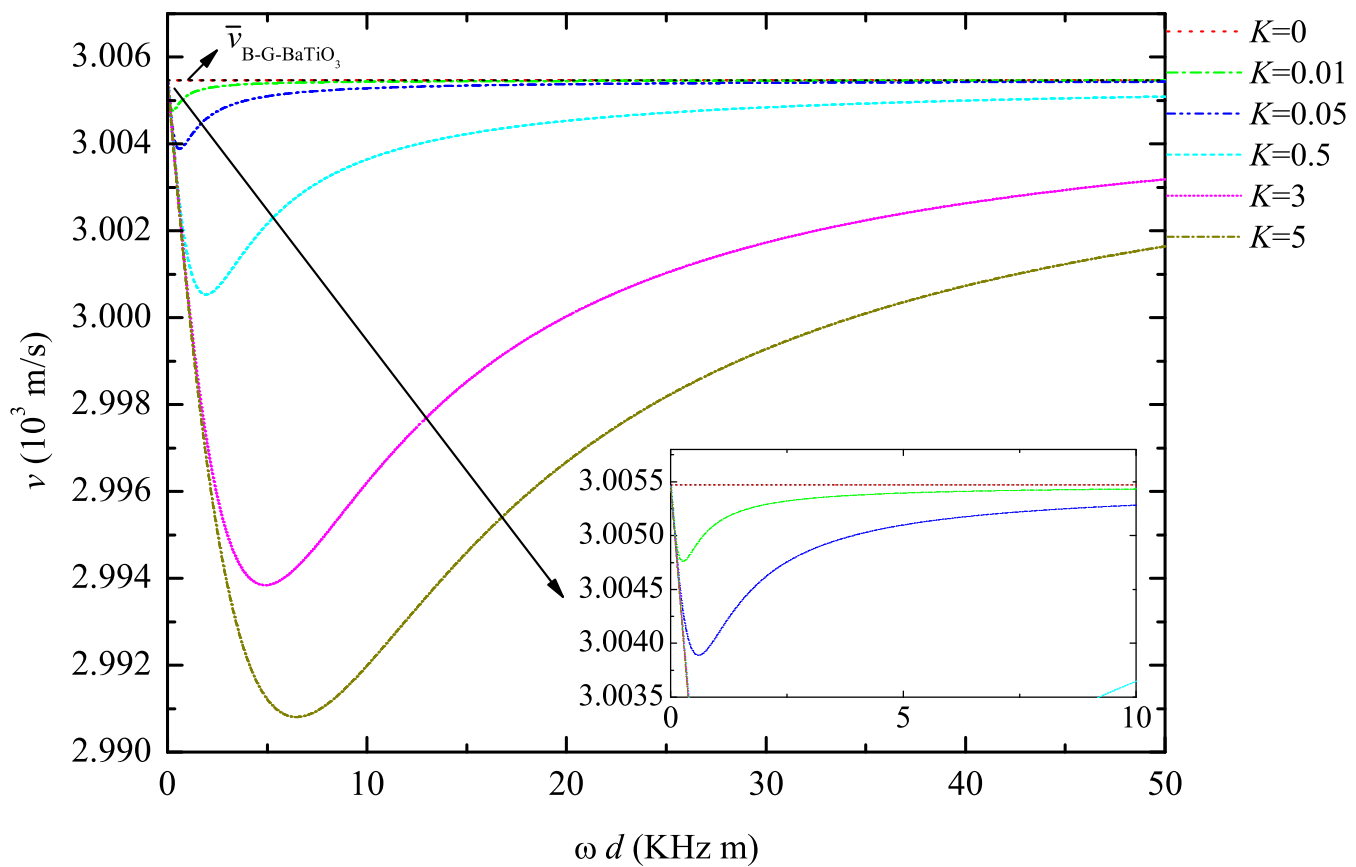

(b)

Figure 3. Influence of imperfect bonding on the dispersion curves for the symmetric modes in a $\mathrm{BaTiO}_{3} / \mathrm{CoFe}_{2} \mathrm{O}_{4} / \mathrm{BaTiO}_{3}$ heterostructure: (a) permeable and (b) absorbent bonding. 


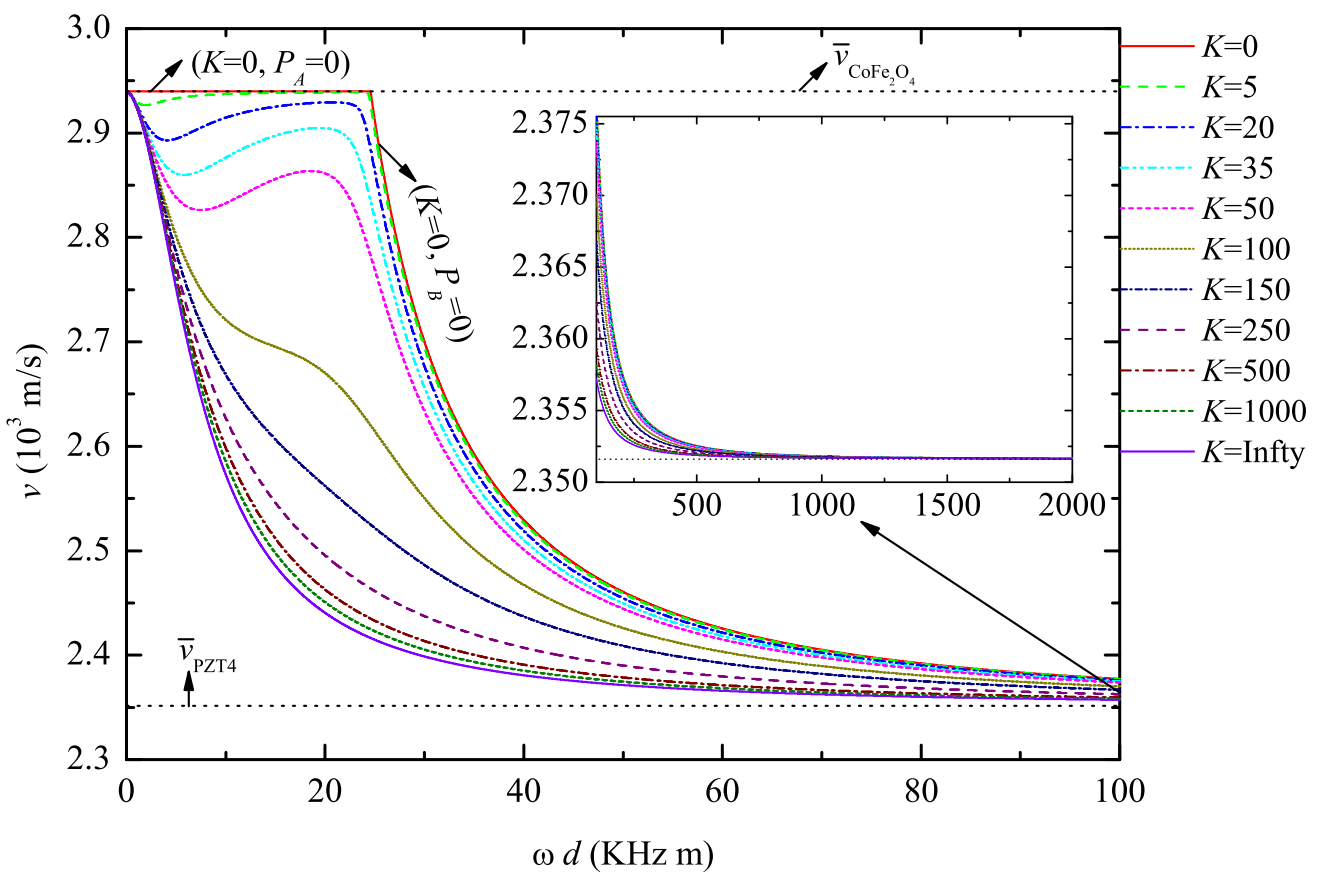

(a)

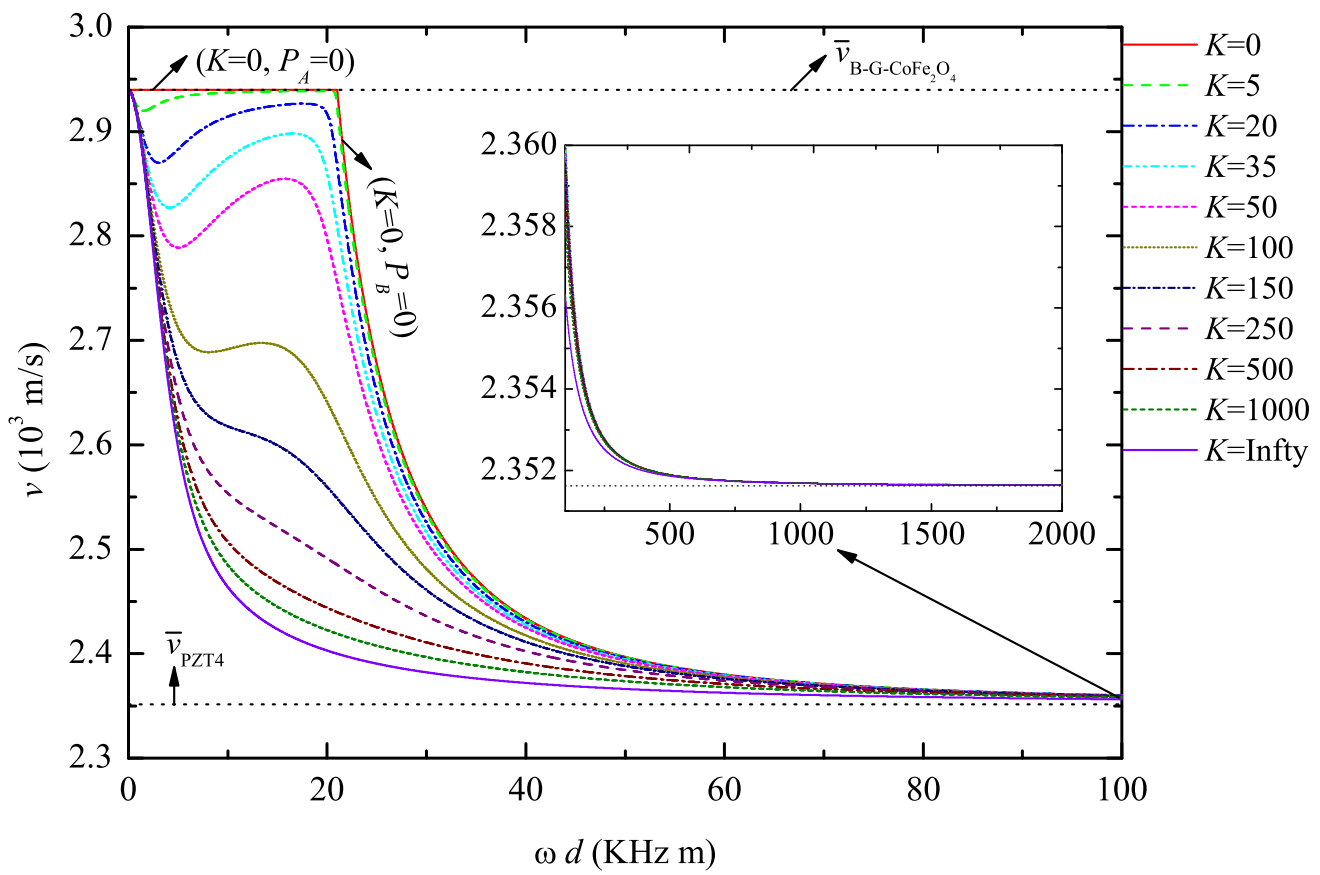

(b)

Figure 4. Influence of imperfect bonding on the dispersion curves for the symmetric modes in a $\mathrm{CoFe}_{2} \mathrm{O}_{4} / \mathrm{PZT} 4 / \mathrm{CoFe}_{2} \mathrm{O}_{4}$ heterostructure: (a) permeable and (b) absorbent bonding. 


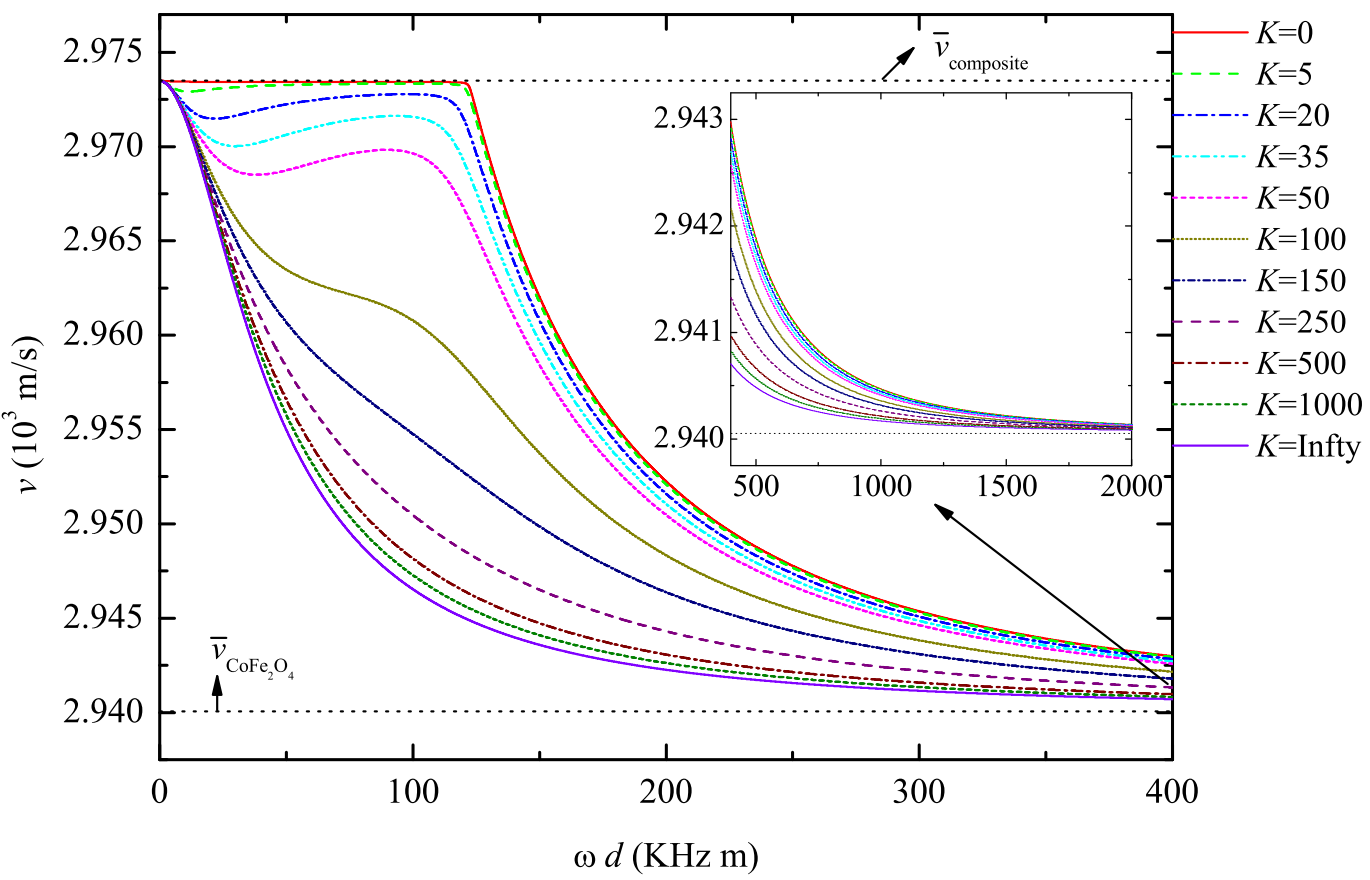

(a)

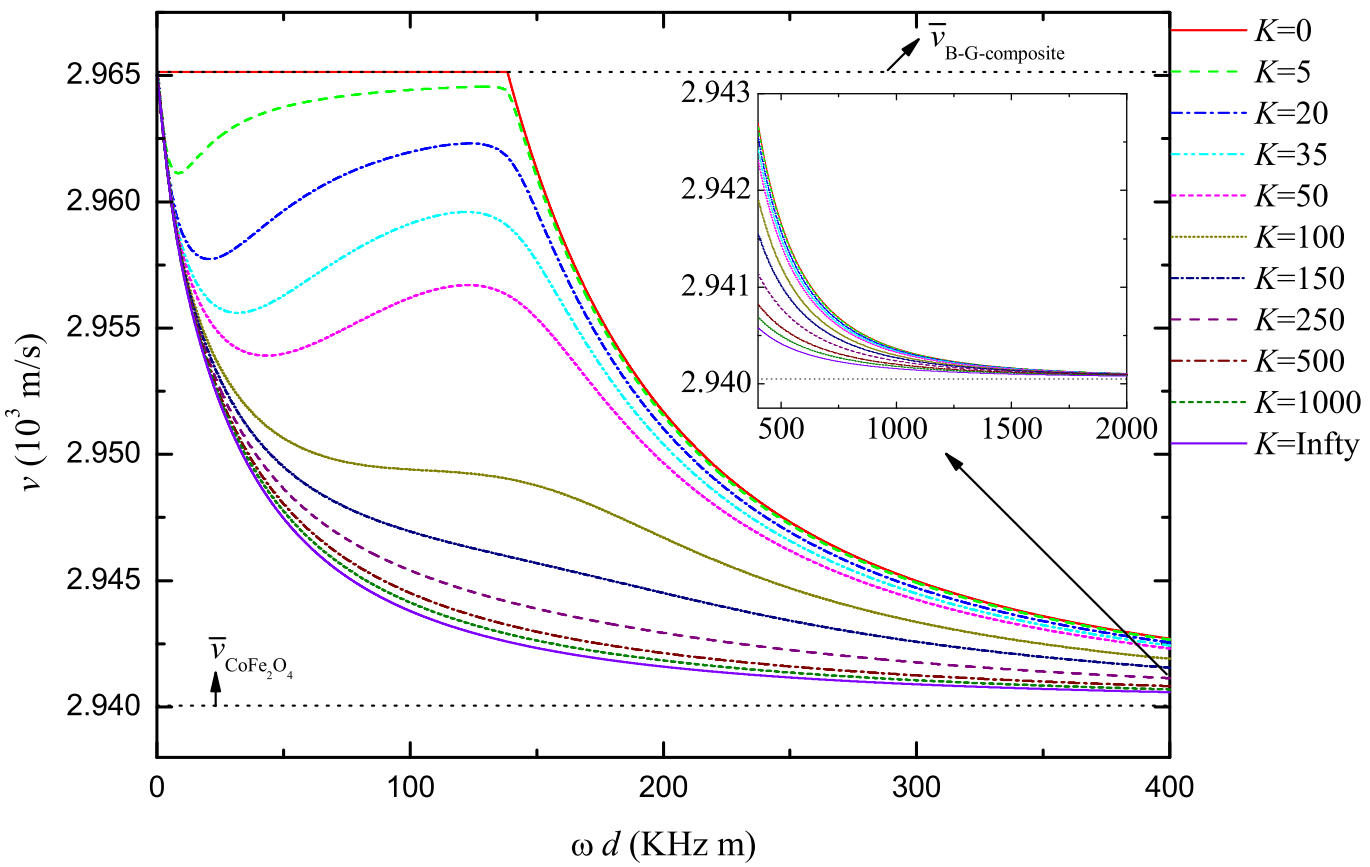

(b)

Figure 5. Influence of imperfect bonding on the dispersion curves for the symmetric modes in a Composite $/ \mathrm{CoFe}_{2} \mathrm{O}_{4} /$ Composite heterostructure: (a) permeable and (b) absorbent bonding. 


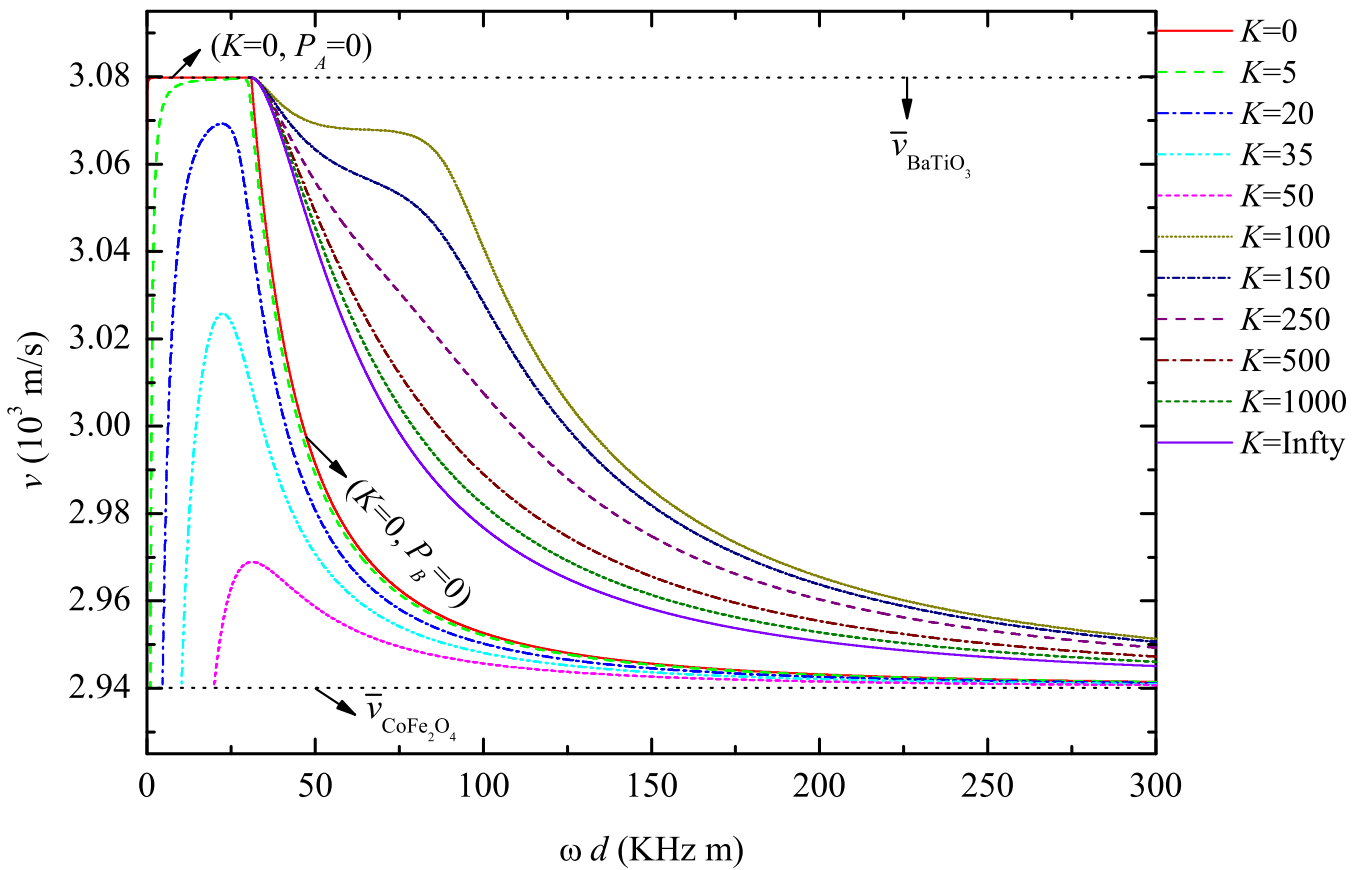

(a)

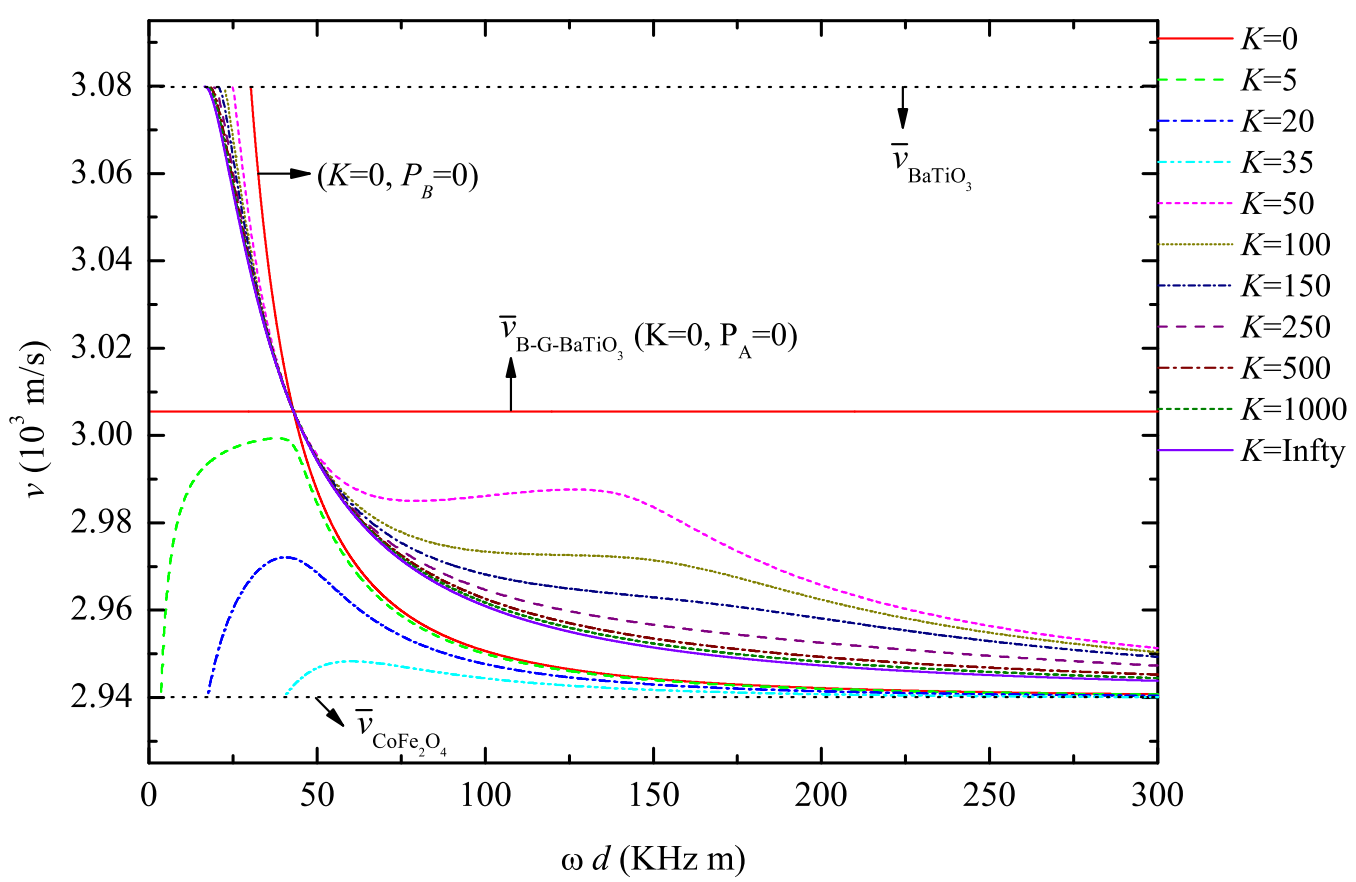

(b)

Figure 6. Influence of imperfect bonding on the dispersion curves for the antisymmetric modes in a $\mathrm{BaTiO}_{3} / \mathrm{CoFe}_{2} \mathrm{O}_{4} / \mathrm{BaTiO}_{3}$ heterostructure: (a) permeable and (b) absorbent bonding. 


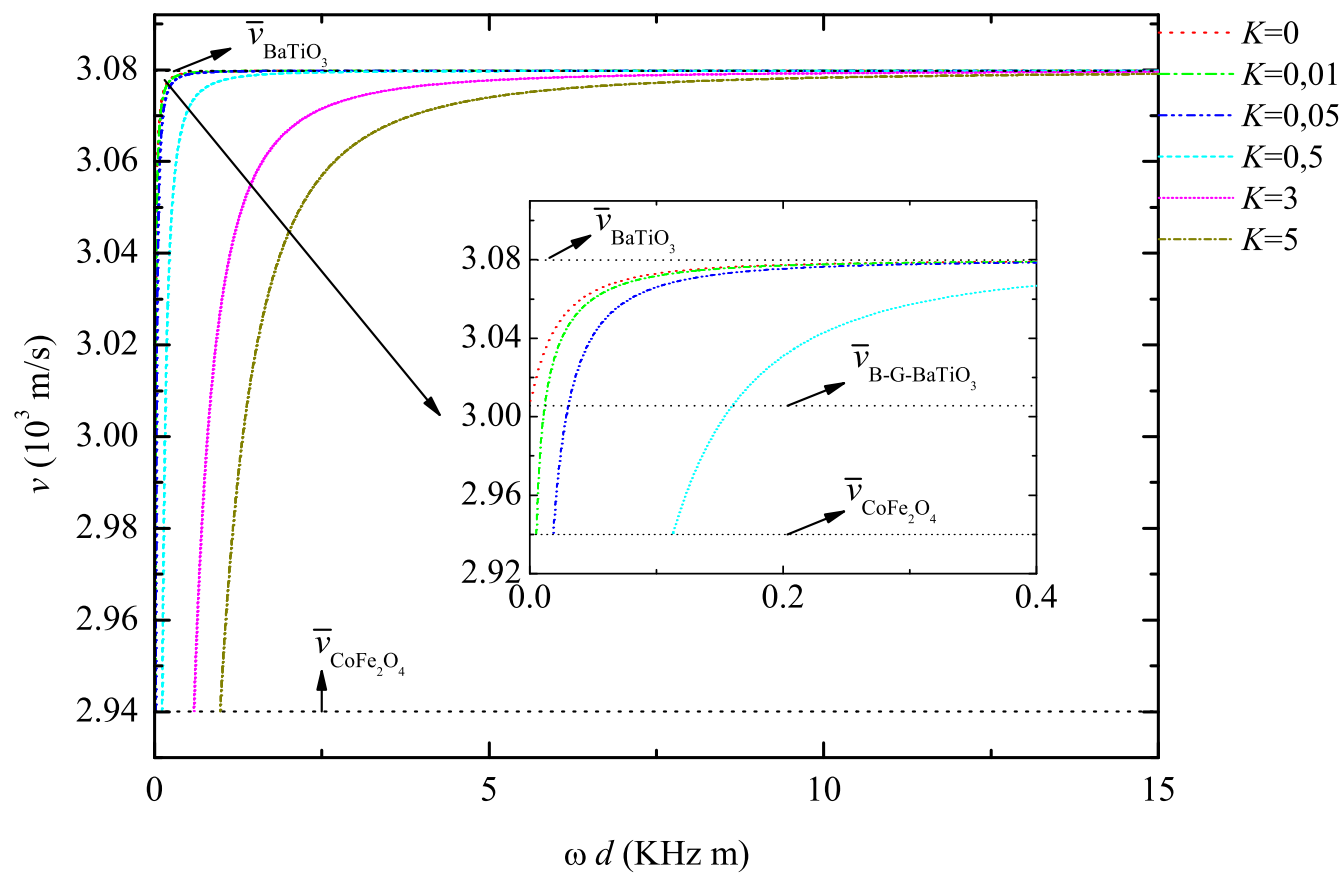

(a)

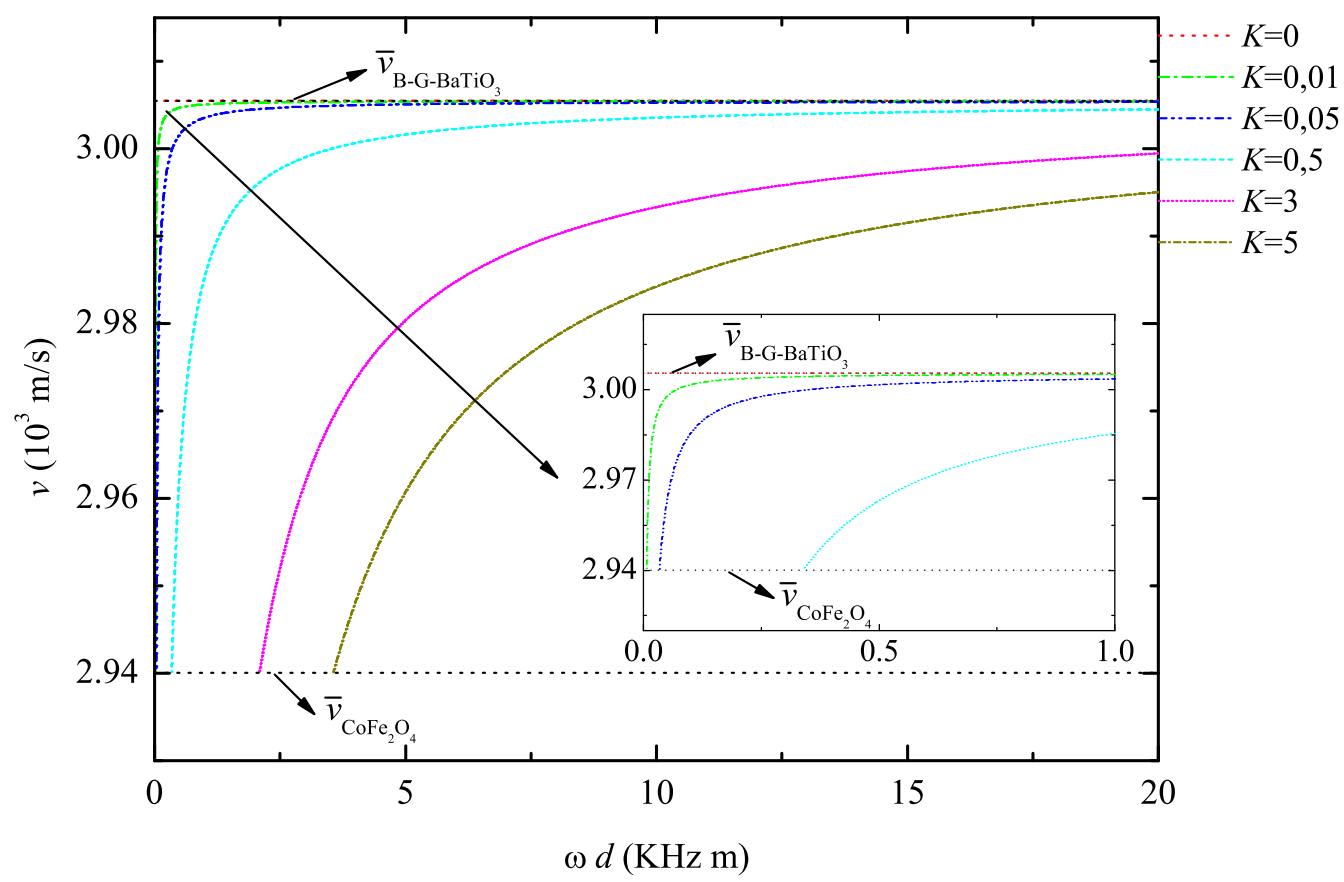

(b)

Figure 7. Influence of imperfect bonding on the dispersion curves for the antisymmetric modes in a $\mathrm{BaTiO}_{3} / \mathrm{CoFe}_{2} \mathrm{O}_{4} / \mathrm{BaTiO}_{3}$ heterostructure: (a) permeable and (b) absorbent bonding. 


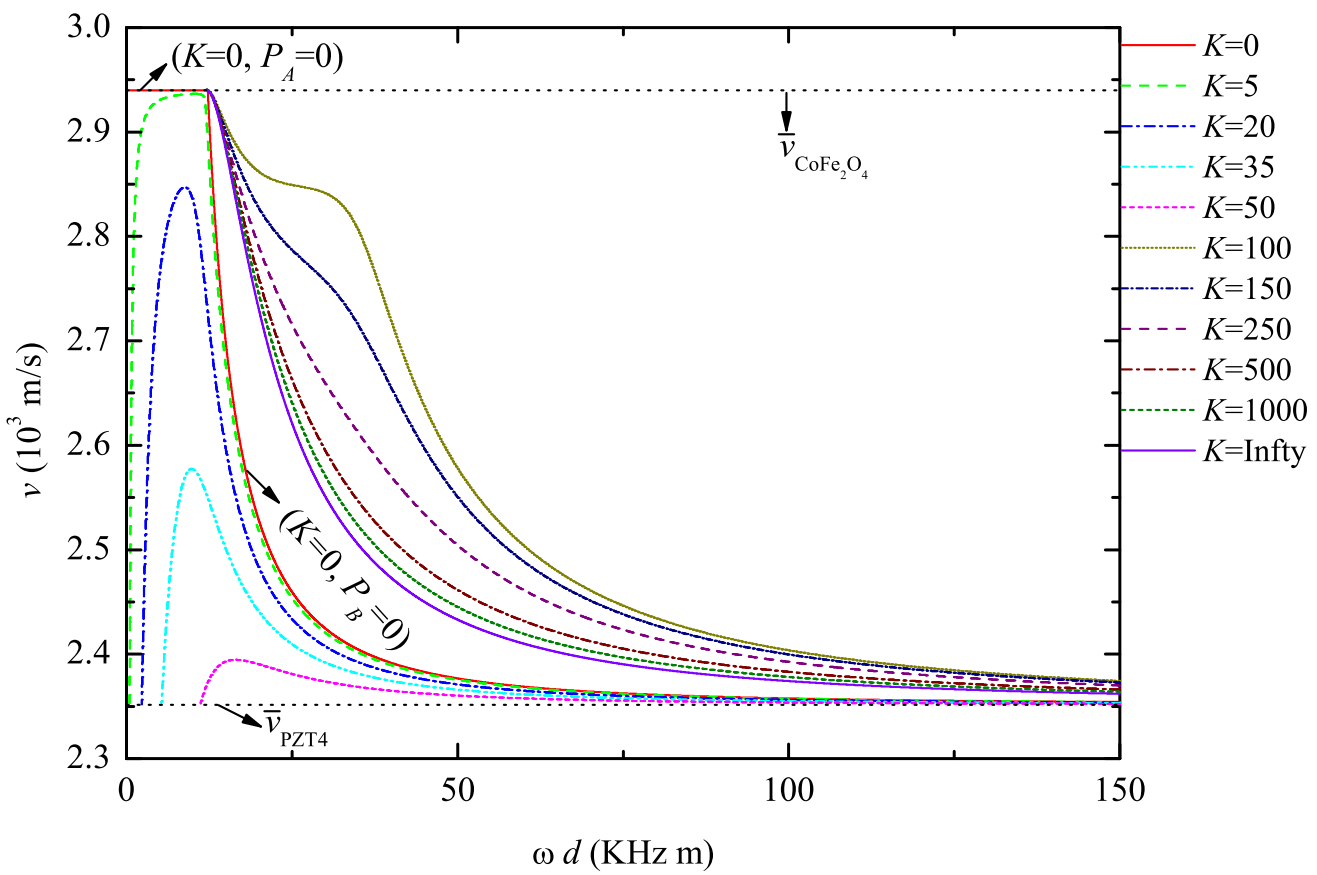

(a)

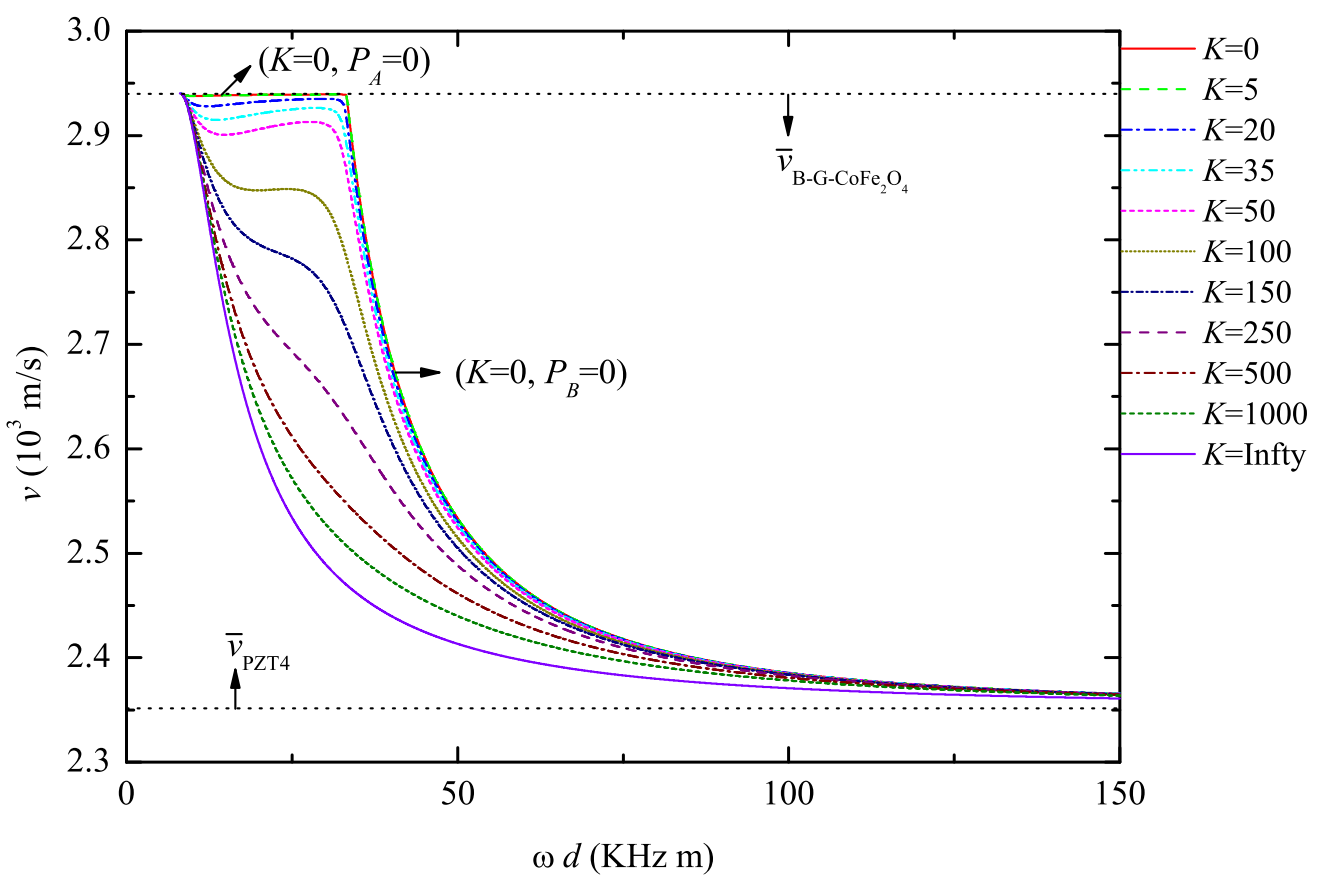

(b)

Figure 8. Influence of imperfect bonding on the dispersion curves for the antisymmetric modes in a $\mathrm{CoFe}_{2} \mathrm{O}_{4} / \mathrm{PZT}_{4} / \mathrm{CoFe}_{2} \mathrm{O}_{4}$ heterostructure: (a) permeable and (b) absorbent bonding. 
not affect the wave propagation at high frequency for a given width $d$. Observe also from Figure 2 a that the velocity $v$ tends to the velocity in phase $\mathrm{A}, \bar{v}_{\mathrm{BaTiO}_{3}}$, as $K$ tends to zero for $\omega d$ less than $62.016 \mathrm{KHz}$.

To analyze Figure 2b, observe from conditions (3-2)b-(3-2)e that there are neither electrical nor magnetic interactions between phases $\mathrm{A}, \mathrm{BaTiO}_{3}$, and $\mathrm{B}, \mathrm{CoFe}_{2} \mathrm{O}_{4}$, across an interface. If $K=0$, recall from (3-2)a that these phases are not bonded at an interface and, therefore, there is no mechanical interaction between them either. In this case, the dispersion relation (4-34) reduces to (4-44), that is, $P_{A} P_{B}=0$. The solution $P_{A}=0$ corresponds to the constant phase velocity of the piezoelectric Bleustein-Gulyaev wave for phase $\mathrm{A}, \mathrm{BaTiO}_{3}$, which is obtained from (4-32), (4-33), and (2-10), and is given by $v_{\mathrm{B}-\mathrm{G}}=\bar{v}_{A} \sqrt{1-e_{A}^{4} /\left(\varepsilon_{A} \bar{c}_{A}^{e}\right)^{2}}$. The dashed straight line in Figure $2 \mathrm{~b}$ corresponds to this case. On the other hand, the solution $P_{B}=0$ corresponds to a dispersive wave for the $\mathrm{CoFe}_{2} \mathrm{O}_{4}$ medium with velocity determined from (4-45). The dashed line at the bottom of Figure $2 b$ corresponds to this case. Observe from this figure that all other dispersion curves are in between the curve for the BleusteinGulyaev wave velocity of the $\mathrm{BaTiO}_{3}$ medium and the curve for the wave velocity of phase $\mathrm{B}$, that is, $\bar{v}_{\mathrm{B}-\mathrm{G}-\mathrm{BaTiO}_{3}}<v<\bar{v}_{\mathrm{CoFe}_{2} \mathrm{O}_{4}}$.

We now consider that $\omega d$ belongs to the interval $(0,50)$ in Figure 2 and show dispersion curves in Figure 3 for small values of $K$ belonging to the set $\{0,0.001,0.005,0.05,3,5\}$. In the permeable case (Figure 3a) the wave velocity $v$ tends to the velocity in phase $\mathrm{A}, \bar{v}_{\mathrm{BaTiO}_{3}}$, as $\omega d$ tends to zero, which is an expected result from (4-18). Note from the graph in the lower right-hand corner, for which $\omega d$ belongs to the interval $(0,10)$, that the wave velocity for $K=0$ is both an upper bound and a limit point to which the wave velocity $v$ tends to as $K$ tends to zero. In the absorbent case (Figure $3 \mathrm{~b}$ ) $v$ tends to the

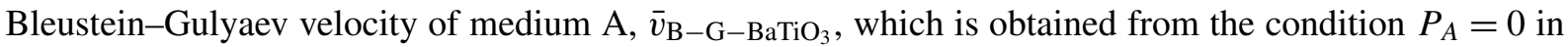
(4-44) and is given by (4-32) together with (4-33). Note from both graphs in Figure 3 that $\bar{v}_{\mathrm{B}-\mathrm{G}-\mathrm{BaTiO}_{3}}$ is also an upper bound and a limit point for $v$ as $K$ tends to zero.

We now show in Figure 4 dispersion curves for the symmetric modes of $\mathrm{SH}$ waves propagating in the heterostructure $\mathrm{CoFe}_{2} \mathrm{O}_{4} / \mathrm{PZT} 4 / \mathrm{CoFe}_{2} \mathrm{O}_{4}$ with either a permeable (Figure 4a) or absorbent (Figure 4b) interface for increasing values of $K$. These curves are qualitatively similar to the curves shown in Figure 2. Thus, all wave velocities tend to the wave velocity in phase $\mathrm{B}, \bar{v}_{\mathrm{PZT} 4}$, as $\omega d \rightarrow \infty$. As $\omega d$ tends to zero, these velocities tend to the wave velocity in phase $\mathrm{A}, \bar{v}_{\mathrm{CoFe}_{2} \mathrm{O}_{4}}$, in Figure $4 \mathrm{a}$ and tend to the BleusteinGulyaev velocity of medium $\mathrm{A}, \bar{v}_{\mathrm{B}-\mathrm{G}-\mathrm{CoFe}_{2} \mathrm{O}_{4}}$, which is given by $\bar{v}_{A} \sqrt{1-f_{A}^{4} /\left(\mu_{A} \bar{c}_{A}^{f}\right)^{2}}$. In Figure 4 all the dispersion curves are in a region bounded by curves corresponding to the cases $\left(K=0, P_{A}=0\right)$, $\left(K=0, P_{B}=0\right)$, and $\bar{v}_{\mathrm{PZT} 4}$.

Next, we show in Figure 5 dispersion curves for the symmetric modes of $\mathrm{SH}$ waves propagating in the heterostructure Composite $/ \mathrm{CoFe}_{2} \mathrm{O}_{4} / \mathrm{Composite}$ with either a permeable (Figure 5a) or absorbent (Figure 5b) interface for increasing values of $K$. We recall from the beginning of this section that composite stands for the composite $\mathrm{BaTiO}_{3} / \mathrm{CoFe}_{2} \mathrm{O}_{4}$ with 30 percent $\mathrm{BaTiO}_{3}$. These curves are qualitatively similar to the corresponding curves in both Figure 2 and Figure 3.

Results for the antisymmetric parts of $\mathrm{SH}$ waves propagating in the heterostructures $\mathrm{BaTiO}_{3} / \mathrm{CoFe}_{2} \mathrm{O}_{4} /$ $\mathrm{BaTiO}_{3}$ and $\mathrm{CoFe}_{2} \mathrm{O}_{4} / \mathrm{PZT} 4 / \mathrm{CoFe}_{2} \mathrm{O}_{4}$ are shown in Figures 6 and 8, respectively, for both types of interface: (a) permeable and (b) absorbent. Observe from Figure 6a that, for the whole range of values of $\omega d$ shown in the figure and for $\bar{v}_{B} \leq v \leq \bar{v}_{A}$, the curve corresponding to both $K=0$ and $P_{B}=0$ is below the curve corresponding to $K=\infty$, there are no other dispersion curves between these two curves, all the curves corresponding to $K \geq 100$ are above the curve corresponding to $K=\infty$, and 
all the curves corresponding to $K \leq 50$ are below the curve corresponding to both $K=0$ and $P_{B}=0$. Similar observations can also be drawn from Figure $8 \mathrm{a}$.

Next, observe from Figure $6 \mathrm{~b}$ that the curves corresponding to, respectively, $\left(K=0, P_{B}=0\right)$ and $K=\infty$ intersect each other at $\left(\bar{v}_{\mathrm{B}-\mathrm{G}-\mathrm{BaTiO}_{3}}, W d\right)=(3.00547,43.02)$. To the left of $\omega d=43.02$ and for $K \geq 50$, the dispersion curves are in between the two curves above. To the right of $\omega d=43.02$ and for $K \geq 50$, these curves are above the curve corresponding to $K=\infty$. In both cases, the curves corresponding to $K \leq 35$ are below all the other curves mentioned above.

In Figure $8 \mathrm{~b}$ observe that all curves intersect at $\left(\bar{v}_{\mathrm{B}-\mathrm{G}-\mathrm{CoFe}_{2} O_{3}}, \omega d\right)=(2.93987,8.32)$. For $\omega d>8.32$, observe also that, contrary to the previous figures, the curve corresponding to $K=\infty$ is below the curve corresponding to $\left(K=0, P_{B}=0\right)$, and that all the other dispersion curves lie in a region bounded by these two curves.

The behavior of the dispersion curves for small values of $K$ in the set $\{0,0.001,0.005,0.05,3,5\}$ and for $0 \leq \omega d \leq 50$ is shown in Figure 7 for the heterostructure $\mathrm{BaTiO}_{3} / \mathrm{CoFe}_{2} \mathrm{O}_{4} / \mathrm{BaTiO}_{3}$. Observe from this figure that the curve corresponding to $K=0$ is above all the other curves and that the wave velocity for $K=0$ tends to the velocity of the Bleustein-Gulyaev wave in phase A as $\omega d \rightarrow 0$.

\section{Conclusions}

The effect of imperfect bonding at the interface of a heterostructure A/B/A with magnetoelectroelastic properties on stationary shear $(\mathrm{SH})$ waves has been investigated. Analytical dispersion relations for absorbent and permeable interfaces are obtained. Several numerical examples for the first branches of the dispersion curves are shown in the range of $\bar{v}_{B}<v<\bar{v}_{A}$. Different limit cases are considered and show a good agreement with corresponding cases reported in recent studies.

Based on numerical examples, the dispersion curves for different values of the material parameter $K$ are shown. The decreasing of the imperfect bonding parameter $K$ decreases the propagation velocity. The dispersion curves for the symmetric modes are bounded between the limit cases $K=0$ and $K=\infty$ for both types of interfaces, permeable and absorbent interfaces. The dispersion curves are confined and not intersecting between the velocity of the outer (A) and the inner (B) media. The symmetric and antisymmetric modes for the limit case $\omega d \rightarrow \infty$ approach asymptotically to $\bar{v}_{B}$ for all values of $K$. These results were obtained numerically for different combinations of materials.

\section{References}

[Alshits et al. 1994] V. I. Alshits, D. M. Barnett, A. N. Darinskii, and J. Lothe, "On the existence problem for localized acoustic waves on the interface between two piezocrystals", Wave Motion 20:3 (1994), 233-244.

[Bleustein 1968] J. L. Bleustein, "A new surface wave in piezoelectric materials", Appl. Phys. Lett. 13:12 (1968), $412-413$.

[Bleustein 1969] J. L. Bleustein, "Some simple modes of wave propagation in an infinite piezoelectric plate", J. Acoust. Soc. Am. 45:3 (1969), 614-620.

[Bravo-Castillero et al. 2008] J. Bravo-Castillero, R. Rodríguez-Ramos, H. Mechkour, J. A. Otero, and F. J. Sabina, "Homogenization of magneto-electro-elastic multilaminated materials", Q. J. Mech. Appl. Math. 61:3 (2008), 311-332.

[Calas et al. 2008] H. Calas, J. A. Otero, R. Rodríguez-Ramos, G. Monsivais, and C. Stern, "Dispersion relations for SH wave in magneto-electro-elastic heterostructures", Int. J. Solids Struct. 45:20 (2008), 5356-5367.

[Chen et al. 2004] W. Q. Chen, J. B. Cai, G. R. Ye, and Y. F. Wang, "Exact three-dimensional solutions of laminated orthotropic piezoelectric rectangular plates featuring interlaminar bonding imperfections modeled by a general spring layer", Int. J. Solids Struct. 41:18-19 (2004), 5247-5263. 
[Chen et al. 2007] J. Chen, E. Pan, and H. Chen, "Wave propagation in magneto-electro-elastic multilayered plates", Int. J. Solids Struct. 44:3-4 (2007), 1073-1085.

[Chen et al. 2008] J. Chen, W. Wang, J. Wang, Z. Yang, and J. Yang, "A thickness mode acoustic wave sensor for measuring interface stiffness between two elastic materials", IEEE Trans. Ultrason. Ferroelectr. Freq. Control 55:8 (2008), 1678-1681.

[Cheng et al. 1996] Z.-Q. Cheng, A. K. Jemah, and F. W. Williams, "Theory for multilayered anisotropic plates with weakened interfaces", J. Appl. Mech. (ASME) 63:4 (1996), 1019-1026.

[Deng 2006] M. Deng, "Analysis of second-harmonic generation of Lamb waves propagating in layered planar structures with imperfect interfaces", Appl. Phys. Lett. 88:22 (2006), 221902.

[Fan et al. 2006a] H. Fan, J. Yang, and L. Xu, "Antiplane piezoelectric surface waves over a ceramic half-space with an imperfectly bonded layer", IEEE Trans. Ultrason. Ferroelectr. Freq. Control 53:9 (2006), 1695-1698.

[Fan et al. 2006b] H. Fan, J. Yang, and L. Xu, "Piezoelectric waves near an imperfectly bonded interface between two halfspaces”, Appl. Phys. Lett. 88:20 (2006), 203509.

[Gulyaev 1969] Y. V. Gulyaev, "Surface electroacoustic waves in solids", J. Exp. Theor. Phys. Lett. 9 (1969), 37-38.

[Handge 2002] U. A. Handge, "Analysis of a shear-lag model with nonlinear elastic stress transfer for sequential cracking of polymer coatings", J. Mater. Sci. 37:22 (2002), 4775-4782.

[Hashin 1991] Z. Hashin, "Thermoelastic properties of particulate composites with imperfect interface", J. Mech. Phys. Solids 39:6 (1991), 745-762.

[Huang and Li 2010] Y. Huang and X. F. Li, "Shear waves guided by the imperfect interface of two magnetoelectric materials", Ultrasonics 50:8 (2010), 750-757.

[Huang and Rokhlin 1992] W. Huang and S. I. Rokhlin, "Interface waves along an anisotropic imperfect interface between anisotropic solids", J. Nondestruct. Eval. 11:3-4 (1992), 185-198.

[Huang et al. 2009] Y. Huang, X.-F. Li, and K. Y. Lee, "Interfacial shear horizontal (SH) waves propagating in a two-phase piezoelectric/piezomagnetic structure with an imperfect interface", Philos. Mag. Lett. 89:2 (2009), 95-103.

[Klarbring and Movchan 1998] A. Klarbring and A. B. Movchan, "Asymptotic modelling of adhesive joints", Mech. Mater. 28:1-4 (1998), 137-145.

[Li and Yang 2006] X.-F. Li and J. S. Yang, "Piezoelectric gap waves between a piezoceramic half-space and a piezoceramic plate", Sens. Actuators A Phys. 132:2 (2006), 472-479.

[Margetan et al. 1992] F. J. Margetan, R. B. Thompson, J. H. Rose, and T. A. Gray, "The interaction of ultrasound with imperfect interfaces: experimental studies of model structures", J. Nondestruct. Eval. 11:3-4 (1992), 109-126.

[Melkumyan 2007] A. Melkumyan, "Twelve shear surface waves guided by clamped/free boundaries in magneto-electro-elastic materials", Int. J. Solids Struct. 44:10 (2007), 3594-3599.

[Melkumyan and Mai 2008] A. Melkumyan and Y.-W. Mai, "Influence of imperfect bonding on interface waves guided by piezoelectric/piezomagnetic composites", Philos. Mag. 88:23 (2008), 2965-2977.

[Pan 2001] E. Pan, "Exact solution for simply supported and multilayered magneto-electro-elastic plates", J. Appl. Mech. (ASME) 68:4 (2001), 608-618.

[Pan and Heyliger 2002] E. Pan and P. R. Heyliger, "Free vibrations of simply supported and multilayered magneto-electroelastic plates", J. Sound Vib. 252:3 (2002), 429-442.

[Rokhlin and Wang 1991] S. I. Rokhlin and Y. J. Wang, "Analysis of boundary conditions for elastic wave interaction with an interface between two solids", J. Acoust. Soc. Am. 89:2 (1991), 503-515.

[Termonia 1990] Y. Termonia, "Fibre coating as a means to compensate for poor adhesion in fibre-reinforced materials", $J$. Mater. Sci. 25:1 (1990), 103-106.

[Wang et al. 2003] J. Wang, L. Chen, and S. Fang, "State vector approach to analysis of multilayered magneto-electro-elastic plates", Int. J. Solids Struct. 40:7 (2003), 1669-1680.

[Wang et al. 2007] B. L. Wang, Y.-W. Mai, and O. P. Niraula, "A horizontal shear surface wave in magnetoelectroelastic materials", Philos. Mag. Lett. 87:1 (2007), 53-58.

[Wu et al. 2002] W. Wu, K. Sadeghipour, K. Boberick, and G. Baran, "Predictive modeling of elastic properties of particulatereinforced composites", Mater. Sci. Eng. A 332:1-2 (2002), 362-370. 
[Yang et al. 2006] J. Yang, Y. Hu, Y. Zeng, and H. Fan, "Thickness-shear vibrations of rotated Y-cut quartz plates with imperfectly bonded surface mass layers", IEEE Trans. Ultrason. Ferroelectr. Freq. Control 53:1 (2006), 241-245.

[Zhong et al. 2009] X.-C. Zhong, X.-F. Li, and K. Y. Lee, "Analysis of a mode-I crack perpendicular to an imperfect interface", Int. J. Solids Struct. 46:6 (2009), 1456-1463.

Received 11 Nov 2010. Accepted 22 Mar 2011.

José A. OTERO: jaotero@icmf.inf.cu

Instituto de Cibernética, Matemática y Física, Calle 15 No. 551, Habana 4, CP 10400, Cuba

HÉCTOR CALAS: hcalass@gmail.com

Instituto de Acústica, Consejo Superior de Investigaciones Científicas, Serrano 144, 28006 Madrid, Spain

REINALDO RODRÍGUEZ-RAMOS: reinaldo@matcom.uh.cu

Facultad de Matemática y Computación, Universidad de La Habana, San Lázaro esq. L, Vedado, Habana 4, CP 10400, Cuba

JULIÁn BRAVO-CASTILLERO: jbravo@matcom.uh.cu

Facultad de Matemática y Computación, Universidad de La Habana, San Lázaro esq. L, Vedado, Habana 4, CP 10400, Cuba

ADAIR R. AgUiAR: aguiarar@sc.usp.br

Department of Structural Engineering, University of São Paulo, Av. Trabalhador Sãocarlense, 400, 13566-590 São Carlos, SP, Brazil

http://www.set.eesc.usp.br/portal/pt/docentes/110-adair-roberto-aguiar

GUILLERMO MONSIVAIS: monsi@fisica.unam.mx

Instituto de Física, Universidad Nacional Autónoma de México, Apdo. Postal 20-364, 01000 México, D.F., México 


\title{
JOURNAL OF MECHANICS OF MATERIALS AND STRUCTURES
}

\author{
jomms.org
}

Founded by Charles R. Steele and Marie-Louise Steele

EDITORS

Charles R. SteEle

DAVIDE BIGONI

Stanford University, USA

YASUHIDE SHINDO

University of Illinois at Urbana-Champaign, USA

Tohoku University, Japan

\section{EDITORIAL BOARD}

$\begin{aligned} \text { H. D. BUI } & \text { École Polytechnique, France } \\ \text { J. P. CARTER } & \text { University of Sydney, Australia } \\ \text { R. M. CHRISTENSEN } & \text { Stanford University, USA } \\ \text { G. M. L. GLADWELL } & \text { University of Waterloo, Canada } \\ \text { D. H. HODGES } & \text { Georgia Institute of Technology, USA } \\ \text { J. HUTCHINSON } & \text { Harvard University, USA } \\ \text { C. HWU } & \text { National Cheng Kung University, Taiwan } \\ \text { B. L. KARIHALOO } & \text { University of Wales, UK } \\ \text { Y. Y. KIM } & \text { Seoul National University, Republic of Korea } \\ \text { Z. MROZ } & \text { Academy of Science, Poland } \\ \text { D. PAMPLONA } & \text { Universidade Católica do Rio de Janeiro, Brazil } \\ \text { M. B. RUBIN } & \text { Technion, Haifa, Israel } \\ \text { A. N. SHUPIKOV } & \text { Ukrainian Academy of Sciences, Ukraine } \\ \text { T. TARNAI } & \text { University Budapest, Hungary } \\ \text { F. Y. M. WAN } & \text { University of California, Irvine, USA } \\ \text { P. WRIGGERS } & \text { Universität Hannover, Germany } \\ \text { W. YANG } & \text { Tsinghua University, China } \\ \text { F. ZIEGLER } & \text { Technische Universität Wien, Austria } \\ & \\ \text { PRODUCTION } & \text { contact@ msp.org } \\ \text { SILVIO LEVY } & \text { Scientific Editor }\end{aligned}$

Cover design: Alex Scorpan

Cover photo: Mando Gomez, www.mandolux.com

See http://jomms.org for submission guidelines.

JoMMS (ISSN 1559-3959) is published in 10 issues a year. The subscription price for 2011 is US \$520/year for the electronic version, and \$690/year (+\$60 shipping outside the US) for print and electronic. Subscriptions, requests for back issues, and changes of address should be sent to Mathematical Sciences Publishers, Department of Mathematics, University of California, Berkeley, CA 94720-3840.

JoMMS peer-review and production is managed by EditFLow ${ }^{\circledR}$ from Mathematical Sciences Publishers.

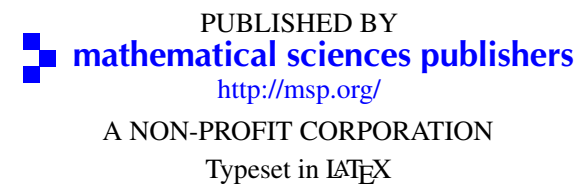

Copyright (C2011 by Mathematical Sciences Publishers 


\section{Journal of Mechanics of Materials and Structures}

\section{Volume 6, No. 7-8}

September-October 2011

\section{Special issue \\ Eleventh Pan-American Congress \\ of Applied Mechanics (PACAM XI)}

Preface

Adair R. Aguiar

949

Influence of specimen geometry on the Portevin-Le Châtelier effect due to dynamic strain aging

for the AA5083-H116 aluminum alloy

Rodrigo Nogueira de Codes and Ahmed Benallal

Dispersion relations for SH waves on a magnetoelectroelastic heterostructure with imperfect

interfaces

J. A. Otero, H. Calas, R. Rodríguez, J. Bravo, A. R. Aguiar and G. Monsivais

Numerical linear stability analysis of a thermocapillary-driven liquid bridge with magnetic stabilization

Yue Huang and Brent C. Houchens

Numerical investigation of director orientation and flow of nematic liquid crystals in a planar 1:4 expansion Pedro a. Cruz, Murilo F. Tomé, IAin W. Stewart and Sean McKee

Critical threshold and underlying dynamical phenomena in pedestrian-induced lateral vibrations of footbridges

Stefano LenCI and LAURA MARCHEgGiani

Free vibration of a simulation CANDU nuclear fuel bundle structure inside a tube

XUAN ZHANG and SHUdONG Yu

Nonlinear dynamics and sensitivity to imperfections in Augusti's model

D. Orlando, P. B. Gonçalves, G. Rega and S. LenCi

Active control of vortex-induced vibrations in offshore catenary risers: A nonlinear normal mode approach

CArlos E. N. MAZzilli and César T. SANCheS

Nonlinear electromechanical fields and localized polarization switching of piezoelectric macrofiber composites

Yasuhide Shindo, Fumio Narita, KoJi SATo and Tomo TAKeda

1089

Three-dimensional BEM analysis to assess delamination cracks between two transversely isotropic materials

Nicolás O. Larrosa, Jhonny E. Ortiz and Adrián P. Cisillino

Porcine dermis in uniaxial cyclic loading: Sample preparation, experimental results and modeling

A. E. Ehret, M. Hollenstein, E. MAzzA and M. Itskov

Analysis of nonstationary random processes using smooth decomposition

Rubens SAMpaio and Sergio Bellizzi

Perturbation stochastic finite element-based homogenization of polycrystalline materials

S. LePage, F. V. Stump, I. H. Kim and P. H. Geubelle

A collocation approach for spatial discretization of stochastic peridynamic modeling of fracture

Georgios I. Evangelatos and POL D. SPANOS 